\title{
Towards a Better Understanding of Cognitive Deficits in Absence Epilepsy: a Systematic Review and Meta-Analysis
}

\author{
Eric L. A. Fonseca Wald ${ }^{1,2,3}$ • Jos G. M. Hendriksen ${ }^{1,2} \cdot$ Gerald S. Drenthen $^{3,4} \cdot$ Sander M. J. V. Kuijk ${ }^{5}$ • \\ Albert P. Aldenkamp ${ }^{1,2,6}$. Johan S. H. Vles ${ }^{1,3} \cdot$ R. Jeroen Vermeulen ${ }^{1,3}$ • Mariette H. J. A. Debeij-van Hall ${ }^{2}$. \\ Sylvia Klinkenberg ${ }^{1,3}$
}

Received: 26 July 2018 / Accepted: 21 October 2019 / Published online: 27 November 2019

(C) The Author(s) 2019

\begin{abstract}
Cognition in absence epilepsy (AE) is generally considered undisturbed. However, reports on cognitive deficits in AE in recent years have suggested otherwise. This review systematically assesses current literature on cognitive performance in children with AE. A systematic literature search was performed in Pubmed, Embase, Cochrane and Web of Science. All studies reporting on cognitive performance in children with AE were considered. In total 33 studies were eligible for inclusion. Neuropsychological tests were classified into the following domains: intelligence; executive function; attention; language; motor \& sensoryperceptual examinations; visuoperceptual/visuospatial/visuoconstructional function; memory and learning; achievement. Random-effect meta-analyses were conducted by estimating the pooled mean and/or pooling the mean difference in casecontrol studies. Full-scale IQ in children with AE was estimated at 96.78 (95\%CI:94.46-99.10) across all available studies and in case-control studies IQ was on average 8.03 (95\%CI:-10.45- -5.61) lower. Verbal IQ was estimated at 97.98 (95\%CI:95.80-100.16) for all studies and 9.01 (95\%CI:12.11- -5.90) points lower in case-control studies. Performance IQ was estimated at 97.23 (93.24-101.22) for all available studies and 5.32 (95\%CI:-8.27-2.36) points lower in case-control studies. Lower performance was most often reported in executive function (cognitive flexibility, planning, and verbal fluency) and attention (sustained, selective and divided attention). Reports on school difficulties, neurodevelopmental problems, and attentional problems were high. In conclusion, in contrast to common beliefs, lower than average neurocognitive performance was noted in multiple cognitive domains, which may influence academic and psychosocial development.
\end{abstract}

Keywords Absence epilepsy $\cdot$ Cognition $\cdot$ Meta-analysis $\cdot$ Systematic review

\section{Introduction}

Typical absence seizures as occurring in childhood absence epilepsy (CAE) and juvenile absence epilepsy (JAE) are characterized by demarcated brief episodes of unconsciousness

Electronic supplementary material The online version of this article (https://doi.org/10.1007/s11065-019-09419-2) contains supplementary material, which is available to authorized users.

Eric L. A. Fonseca Wald eric.fonsecawald@mumc.nl

Sylvia Klinkenberg

s.klinkenberg@mumc.nl

1 Department of Neurology, Maastricht University Medical Center+, 6202, AZ Maastricht, The Netherlands

2 Epilepsy Center Kempenhaeghe, Heeze, The Netherlands with generalized $\sim 3-\mathrm{Hz}$ spike-and-wave complexes, visible on an electroencephalogram (EEG), in otherwise healthy children (Guerrini, 2006; Myers \& Fecske, 2016; Panayiotopoulos, 2001; Tenney \& Glauser, 2013). A clear delineation of the clinical spectrum between CAE and JAE

3 School for Mental Health and Neuroscience, Maastricht University, Maastricht, The Netherlands

4 Department of Radiology and Nuclear Medicine, Maastricht University Medical Center+, Maastricht, The Netherlands

5 Department of KEMTA, Maastricht University Medical Center+, Maastricht, The Netherlands

6 Department of Electrical Engineering, Eindhoven University of Technology, Eindhoven, The Netherlands 
is challenging (Hughes, 2009; Tenney \& Glauser, 2013; Trinka et al., 2004). CAE occurs mostly between 4 and 10 years of age whereas JAE occurs between 10 and 17 years of age. Furthermore, JAE is characterized by less frequent absence seizures, a higher incidence of tonic-clonic seizures and a higher drug dependency during adulthood.

Despite absence epilepsy (AE) being defined a "benign" disorder, a high rate of initial treatment failure, associated therapeutic side effects, development of generalized tonicclonic seizures and psychosocial co-morbidities in more recent reports emphasize its real burden (Bouma, Westendorp, van Dijk, Peters, \& Brouwer, 1996; Caplan et al., 2008; Cnaan et al., 2017; Glauser et al., 2010; IJff et al., 2016; Loughman, Bendrups, \& D'Souza, 2016; Masur et al., 2013).

Starting from early descriptions, cognitive performance in AE has been considered to be normal (Adie, 1924; Currier, Kooi, \& Saidman, 1963). However, in a large randomized clinical trial intelligence was lower than normal values, but still within normal range (Masur et al., 2013). Nevertheless, $36 \%$ of drug-naïve patients presented with attentional deficits. Moreover, subsequent allocation to monotherapy with Valproate was associated with more attentional dysfunction, than allocation to monotherapy with either Ethosuximide or Lamotrigine (Cnaan et al., 2017; Glauser et al., 2013; Glauser et al., 2010). Nevertheless, attentional deficits persisted independent of the allocated anti-epileptic drug treatment or seizure control after 1-year of follow-up (Cnaan et al., 2017; Glauser et al., 2013; Masur et al., 2013). These are intriguing findings, as apart from therapeutic side effects and (inter)ictal activity other underlying mechanisms may also affect cognition in AE (Aldenkamp \& Arends, 2004; Jafarian et al., 2015; Lenck-Santini \& Scott, 2015; Nicolai et al., 2012).

The number of studies reporting on cognitive performance in AE has been growing steadily and warrants a formal review (Berg, Caplan, \& Hesdorffer, 2011; Berg, Levy, Testa, \& Blumenfeld, 2014; Caplan et al., 2008; Cerminara et al., 2013; Cheng et al., 2017; Conant, Wilfong, Inglese, \& Schwarte, 2010; Conde-Guzon \& Cancho-Candela, 2012; Covanis, Skiadas, Loli, Lada, \& Theodorou, 1992; D'Agati, Cerminara, Casarelli, Pitzianti, \& Curatolo, 2012; Fastenau et al., 2009; Franzoni et al., 2015; Gencpinar et al., 2016; Guerrini et al., 2015; Henkin et al., 2003; Henkin et al., 2005; Kernan et al., 2012; Levav et al., 2002; Lopes, Monteiro, Fonseca, Robalo, \& Simoes, 2014; Lopes et al., 2013; Masur et al., 2013; Mostafa, Talaat, Shalaby, ElFayoumy, \& Labib, 2014; Nolan et al., 2004; Oostrom et al., 2003; Pavone et al., 2001; Schraegle, Nussbaum, \& Stefanatos, 2016; Shinnar et al., 2017; Sinclair \& Unwala, 2007; Siren et al., 2007; Talero-Gutierrez, Sanchez-Torres, \& Velez-van-Meerbeke, 2015; Urena-Hornos et al., 2004; Vanasse, Beland, Carmant, \& Lassonde, 2005; Vega et al., 2010; Verrotti et al., 2011; Wirrell, Camfield, Camfield, Gordon, \& Dooley, 1996). A previous meta-analysis in idiopathic generalized epilepsy found significant impairments in general cognitive ability and across a wide variety of other cognitive domains (Loughman, Bowden, \& D'Souza, 2014). Sub-analyses were available for CAE, however, only four studies were included. Therefore, our review is aimed specifically to address all studies on cognitive performance in AE. Although, the different syndromes in idiopathic generalized epilepsy may constitute a biological continuum, and therefore resemble in their neuropsychological profile, specific cognitive deficits may still exist. Knowledge on cognitive deficits in AE may help clinician's establish better neuropsychological batteries and direct precautionary measures, as cognitive deficits may go unnoticed by their surroundings (Masur et al., 2013). Therefore, this review systematically assesses current literature on cognitive performance in children with $\mathrm{AE}$.

\section{Methods}

This review adheres to the Preferred Reporting Items for Systematic Reviews and Meta-analysis guidelines (PRISMA) (Moher, Liberati, Tetzlaff, \& Altman, 2009). The methods and procedures for this review are available in the paper with additional information provided in the supplemental materials. The review was not registered prior to conducting this review.

\section{Selection Eligibility}

Selection criteria were defined according to PICOS: Participants $=$ Children with $\mathrm{AE}$, either defined as $3-4 \mathrm{~Hz}$ spike-wave complexes or a syndromic classification of CAE and/or JAE; Intervention/diagnostic $=$ neuropsychological tests (batteries) or reports on school performance; Comparison $=$ normative values (for example reports using standardized scores as these represents scores relative to the normative sample of the test) or a control group; Outcome = neuropsychological function based reported as a median/ average score or prevalence of impairment based on cut-off values or a direct comparison of test scores with a control group; Design $=$ Observational studies (cohort studies/casecontrol studies) or clinical trials (depending of the design of the clinical trial these data are also regarded as observational data, for example baseline neuropsychological results).

\section{Systematic Literature Search}

The search strategy consisted of indexed terms and free text words on absence epilepsy in combination with terms on observational research and clinical trials (the search in Pubmed is provided as a Supplemental file). The following electronic databases were searched: Pubmed (until 29-09-2017), EMBASE (until 23-03-2017), Cochrane (until 05-04-2017) 
and Web of Science (all databases) (until 06-04-2017). Furthermore, references of included articles were hand searched to find additional relevant articles. In case multiple publications were available on the same study sample or if reports reported overlapping results, only the most recent data were used. Only studies written in English, Dutch or Spanish were considered. Firstly, titles and/or abstracts identified by the search were first screened by EFW to remove any unrelated hits. Secondly, the remaining abstracts were screened by EFW and another author (GSD or SK or MD or JH) based on the predefined selection criteria. The second researcher was blinded for journal, authors, title, date of publication and publication language. Any discrepancies were resolved by consensus or by screening the full-text subsequently. Eligibility assessment of full-text articles was performed by EFW and one of the neurologists (MD or SK). Any discrepancies were resolved by consensus or by consulting a third author.

\section{Data Extraction}

The following data were extracted: year of publication, country, study design, inclusion/exclusion criteria, number of included patients, number of patients per cognitive assessment, age at onset, age at the time of the study, anti-epileptic drug use and neuropsychological tests results.

Neuropsychological test results were classified to cognitive domains according to Baron and secondly according to the authors of the study or consensus within our team (Baron, 2004). We distinguished the following cognitive domains: intelligence; executive function; attention, language; motor and sensory-perceptual examinations; visuoperceptual/visuospatial/visuoconstructional function; learning and memory. Additionally, we included results on: achievement tests, parent/teacher (by proxy) reports on attention or attentional deficiency disorders; reported prevalence's of school difficulties; neuropsychological and/or neurodevelopmental problems.

\section{Risk of Bias in Individual Studies}

Risk of bias was evaluated using a modified version of the Newcastle Ottawa Scale made suitable for this review (available in the Supplementary Materials). Two authors (EFW \& SK) independently rated each study, and any disagreement was resolved by consensus. For the interpretation of the total scores we used the following cut-off values as used previously in a systematic review (Marengoni et al., 2018). Scores $>7$ were considered a low risk of bias; 5 to 7 , a moderate risk; and $<5$, a high risk. For the meta-analysis sensitivity analyses were carried out by excluding studies with a score lower than seven.

\section{Meta-Analyses}

Statistical analyses were conducted in R using the "meta" package (version 4.8-2) and "metaphor" package (version 1.9-9) (Schwarzer, 2007; Viechtbauer, 2010). Single-arm meta-analyses (a weighted pooling of the reported means including studies without a control group) were performed by estimating the weighted mean using a random effect model. For this, we used the reported mean and calculated the standard error (SE) for each study. In case a z-score with a 95\% confidence interval was reported we calculated the standard score and its standard deviation. The estimated mean was considered significantly different from normal if the $95 \%$ confidence interval did not include the normative mean of the neuropsychological test.

In addition, a random-effects meta-analysis on the mean difference between cases and controls was performed. Studies that did not report usable data for pooling of results were discarded for the meta-analyses but were included in this systematic review. Pooling of results was conducted per neuropsychological test if methodology and reporting of the results allowed a direct comparison between studies. $P$ values of $\leq 0.05$ were considered to infer statistical significance.

The presence of small study effect and/or publication bias was assessed by visually inspecting funnel plots for asymmetry. In case of potential outliers, a sensitivity analysis was performed by recalculating the effect size after removal of these studies. Tau-squared $\left(T^{2}\right)$ was used to estimate the true variance of the true effect sizes (Borenstein, Higgins, Hedges, $\&$ Rothstein, 2017). In addition, $\mathrm{I}^{2}$ is reported for descriptive purposes. Due to the limited amount of studies in the metaanalyses we were not able to perform subsequent metaregressions to examine the impact of moderator variables (Shuster, 2011; Thompson \& Higgins, 2002).

\section{Results}

\section{Selection of Studies}

The study selection process is depicted in Fig. 1. A total of 3833 individual articles across all electronic databases were screened. Additionally, nine references from selected articles were screened for eligibility. A total of 506 abstracts were selected for further reading, after which 351 were selected for full-text screening. In the end, 33 articles were included in this systematic review.

\section{Study Characteristics}

Study characteristics of the 33 included articles are listed in Table 1. Eight articles reported on overlapping cohorts, but were included as each article provided complementary data 


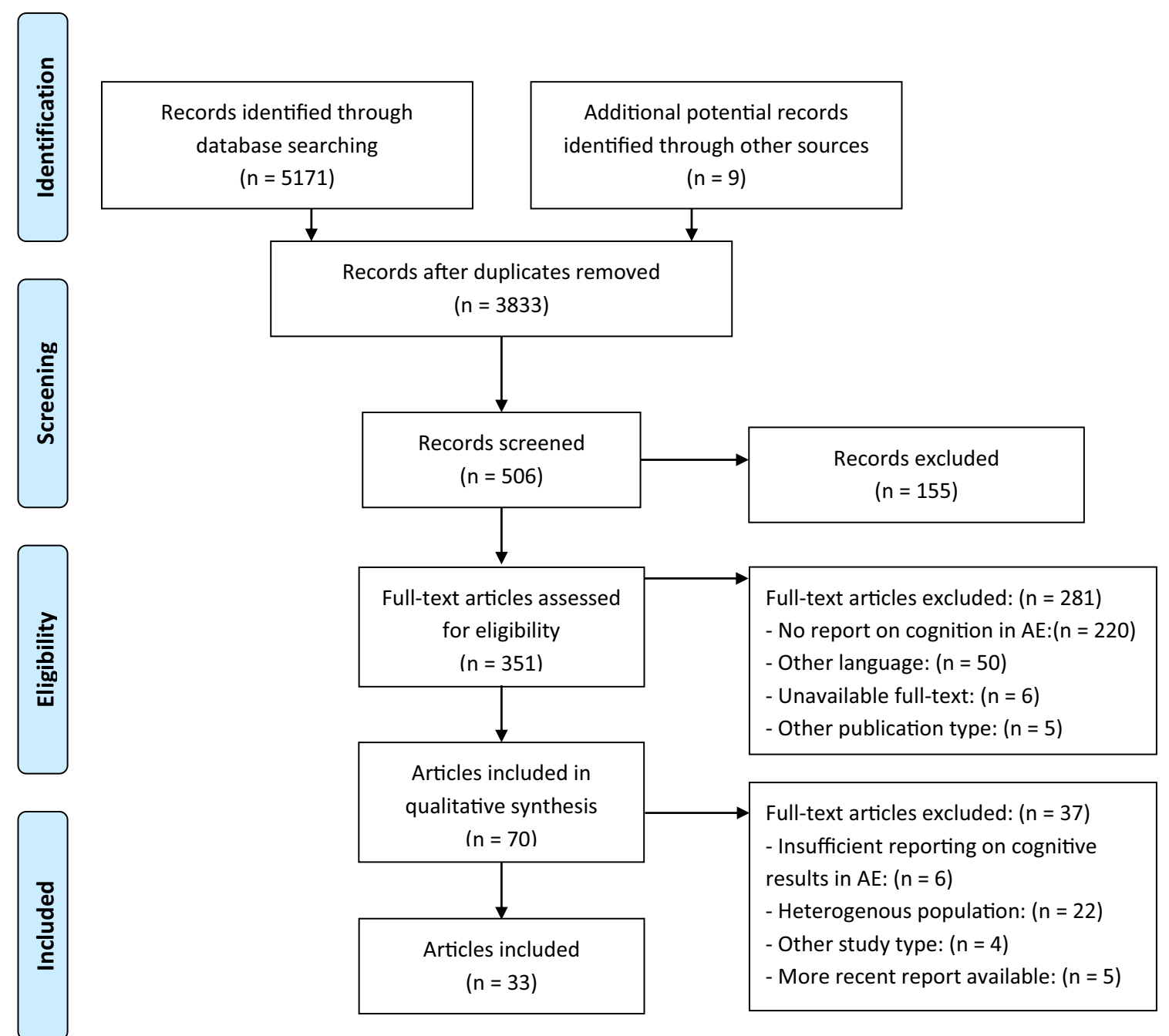

Fig. 1 Flowchart (adapted from Moher et al. (2009))

in that cohort (Berg et al., 2011; Berg et al., 2014; Henkin et al., 2003; Henkin et al., 2005; Lopes et al., 2014; Lopes et al., 2013; Masur et al., 2013; Shinnar et al., 2017). In total, data were available for 29 samples. In total, 17 cohorts reported only on CAE (Berg et al., 2011; Berg et al., 2014; Caplan et al., 2008; Cerminara et al., 2013; Cheng et al., 2017; Conant et al., 2010; D'Agati et al., 2012; Gencpinar et al., 2016; Guerrini et al., 2015; Kernan et al., 2012; Levav et al., 2002; Lopes et al., 2014; Lopes et al., 2013; Masur et al., 2013; Mostafa et al., 2014; Nolan et al., 2004; Oostrom et al., 2003; Schraegle et al., 2016; Shinnar et al., 2017; Siren et al., 2007; Talero-Gutierrez et al., 2015; Vega et al., 2010; Wirrell et al., 1996) and in 12 cohorts reports were available in children with AE or a combination of CAE with JAE (CondeGuzon \& Cancho-Candela, 2012; Covanis et al., 1992; Fastenau et al., 2009; Franzoni et al., 2015; Henkin et al., 2003; Henkin et al., 2005; Oostrom et al., 2003; Pavone et al., 2001; Sinclair \& Unwala, 2007; Siren et al., 2007; Talero-Gutierrez et al., 2015; Urena-Hornos et al., 2004; Vanasse et al., 2005; Verrotti et al., 2011). No studies were available reporting on JAE exclusively. Study sample sizes varied from 10 to 446 , with a mean of 54 participants. The time of cognitive assessment differed from early assessment close to the time of diagnosis to years after diagnosis. Overall Valproic Acid (VPA) seems the most used anti-epileptic drug in the studies. However, some studies only reported the amount of anti-epileptic drug (AED) use (monotherapy, polytherapy) without stating the number of patients on particular AEDs.

Based on our assessment for the risk of bias eighteen studies had a low risk of bias and eleven studies had a moderate risk of bias. No studies were classified to have a high risk of bias. The highest risk of bias was due to inadequate selection and/or description of the non-exposed cohort and a small sample size.

\section{Intelligence}

Table 2 displays the neuropsychological results of the included study per cognitive domain. Wechsler Intelligence Test for 


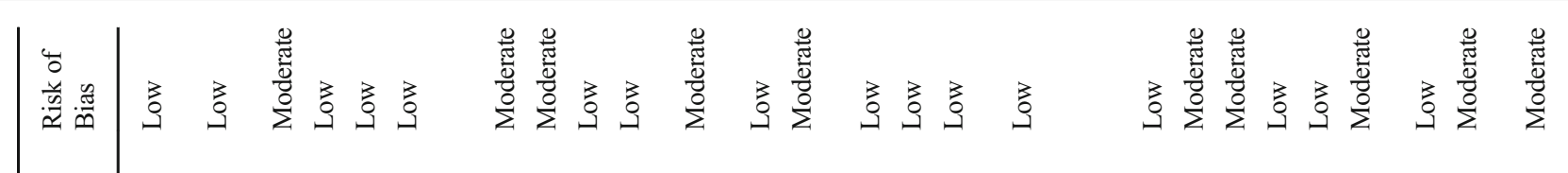

$\sum_{\substack{n \\ m}}^{\infty}$

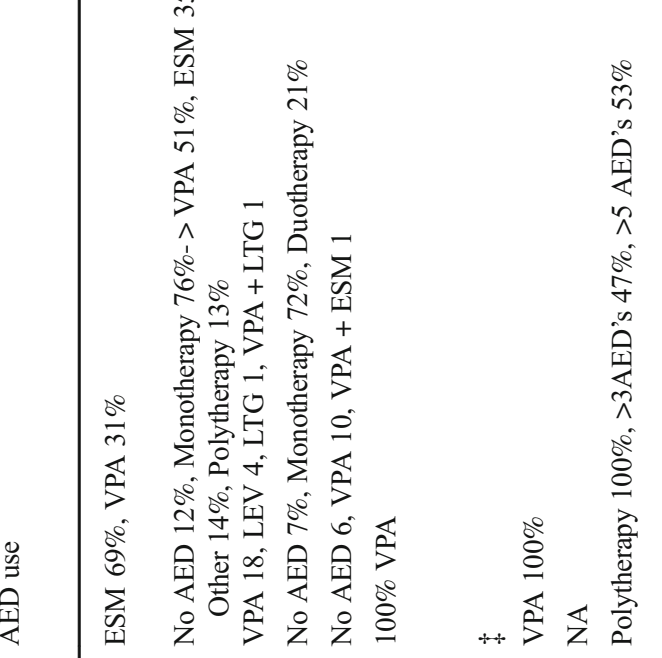

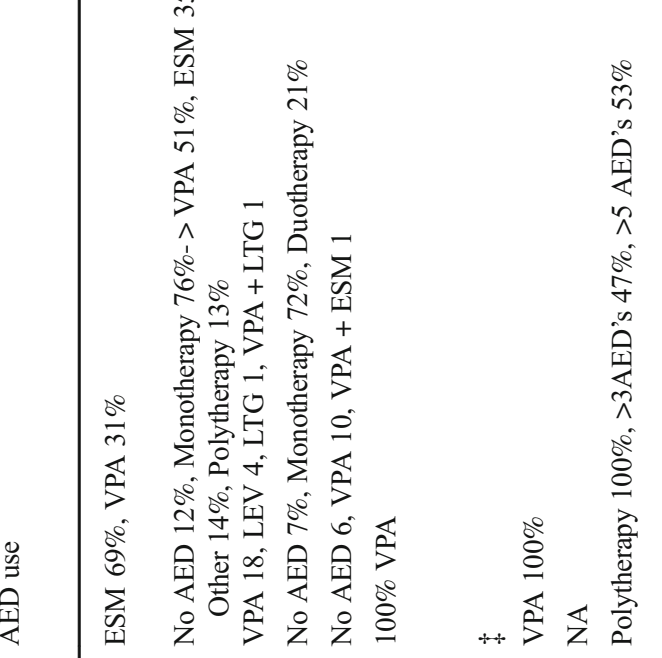

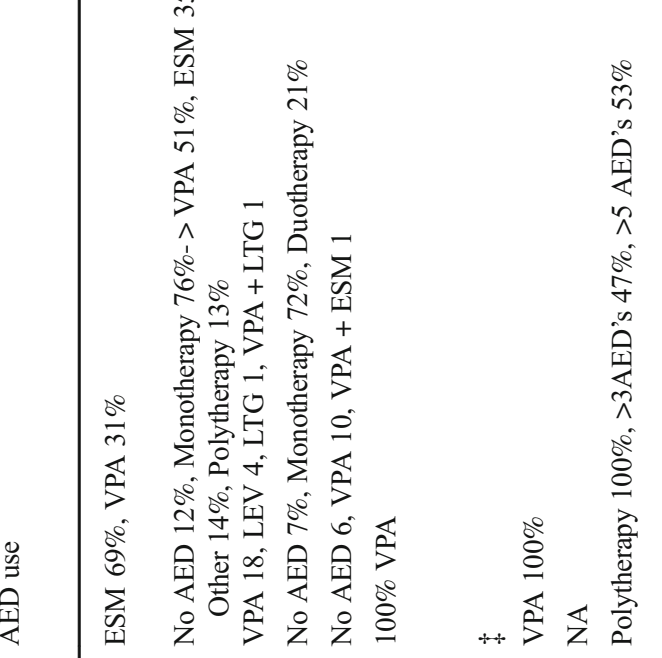

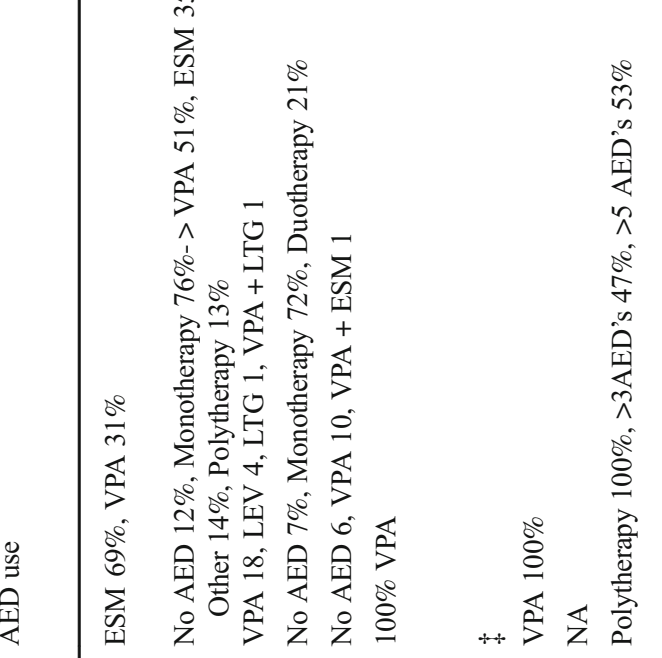

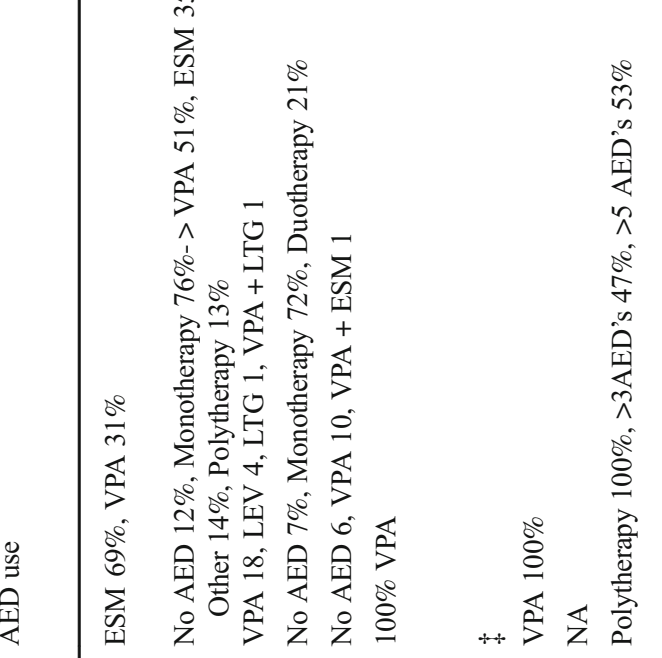

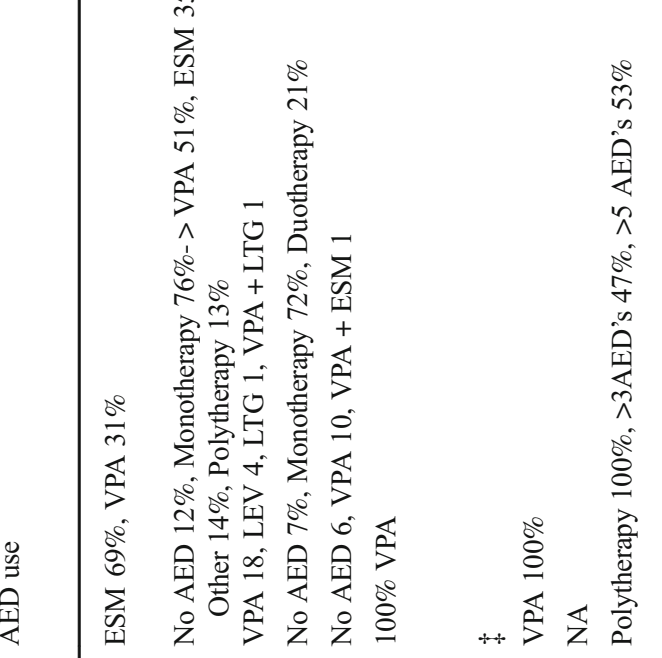

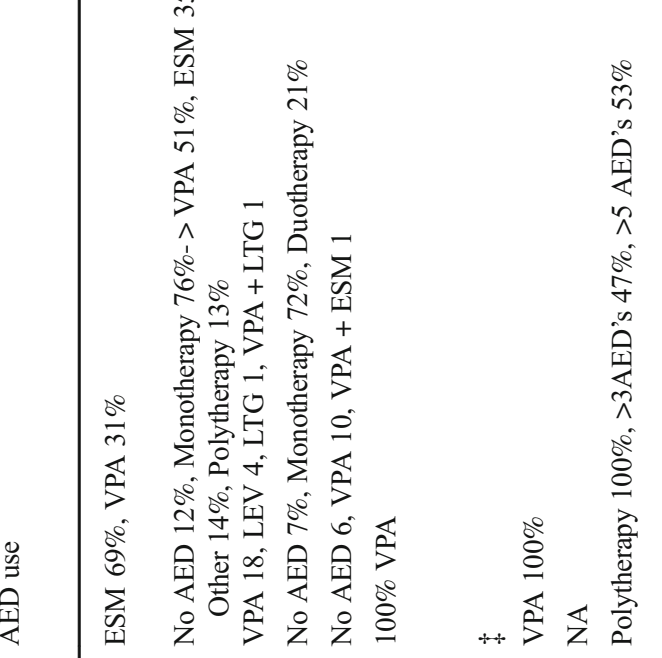

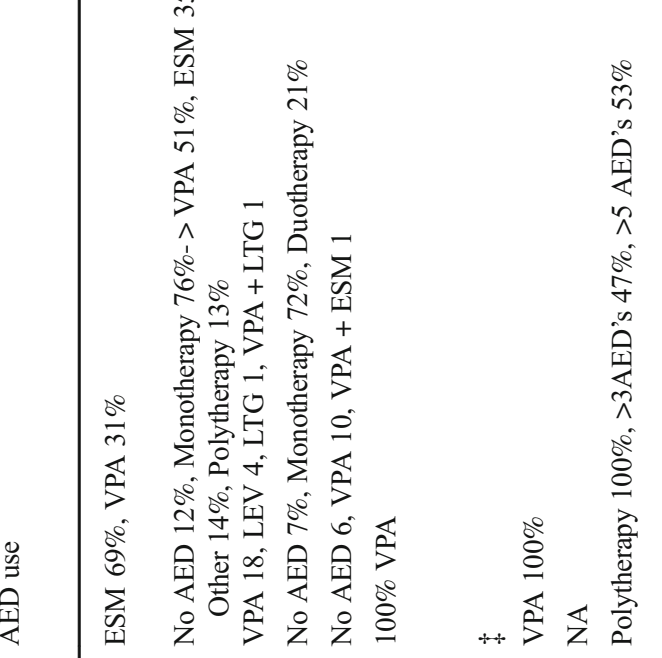

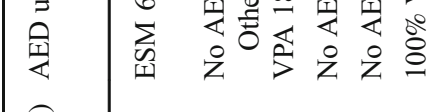

官

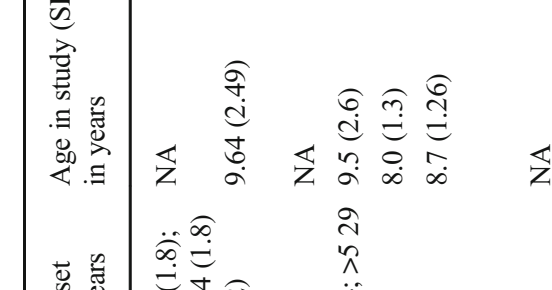

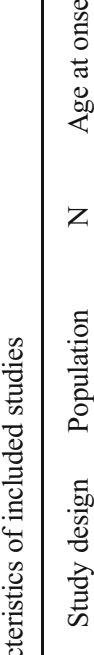

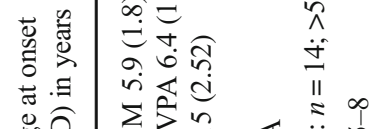

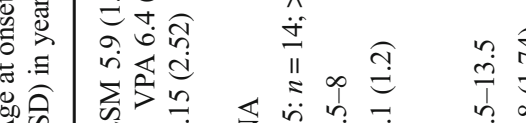

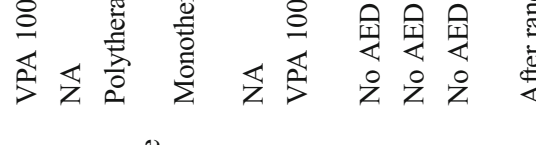

ते

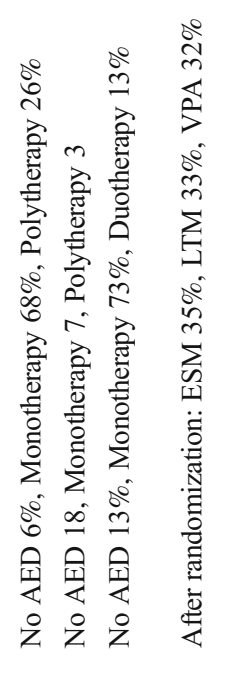<smiles>C1CCC1</smiles>

so

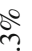

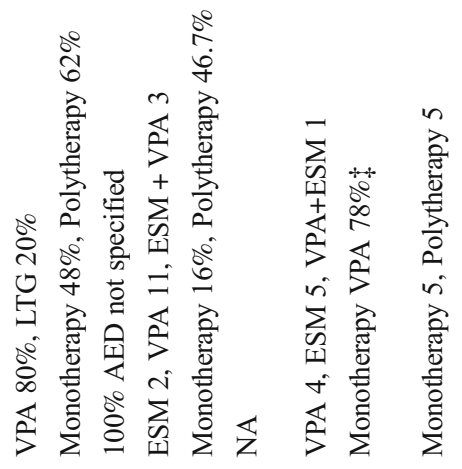

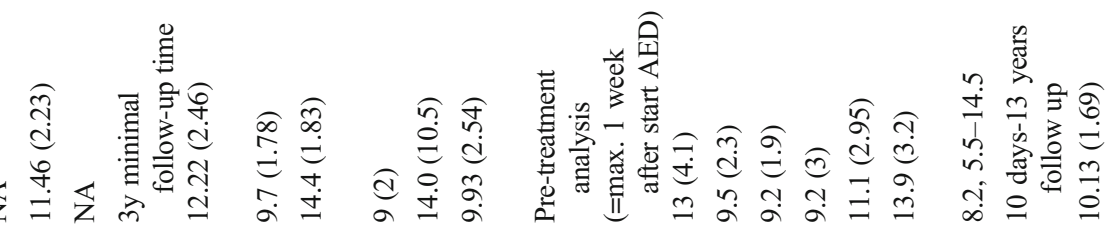

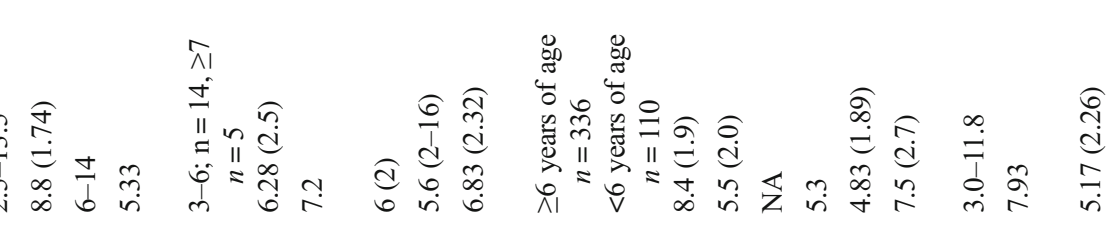

in a ป

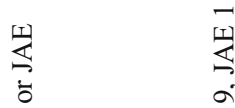

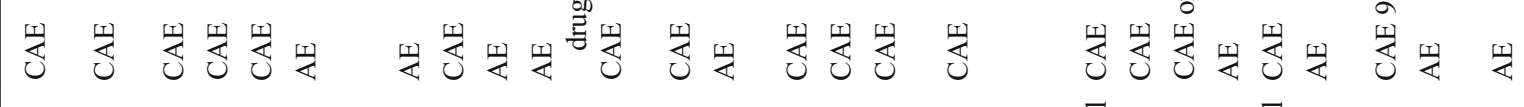

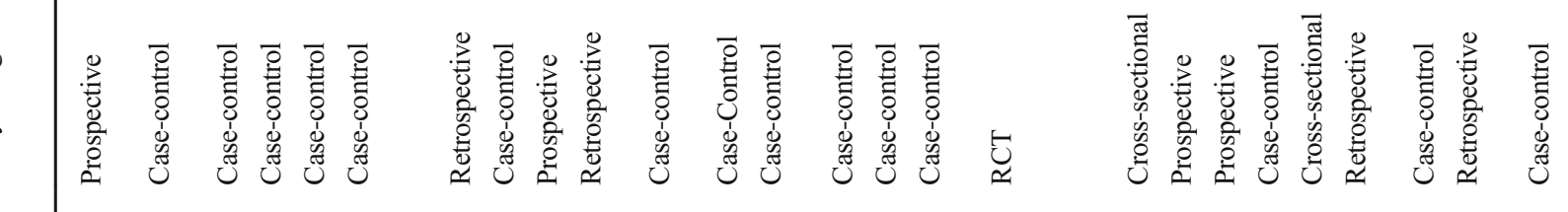

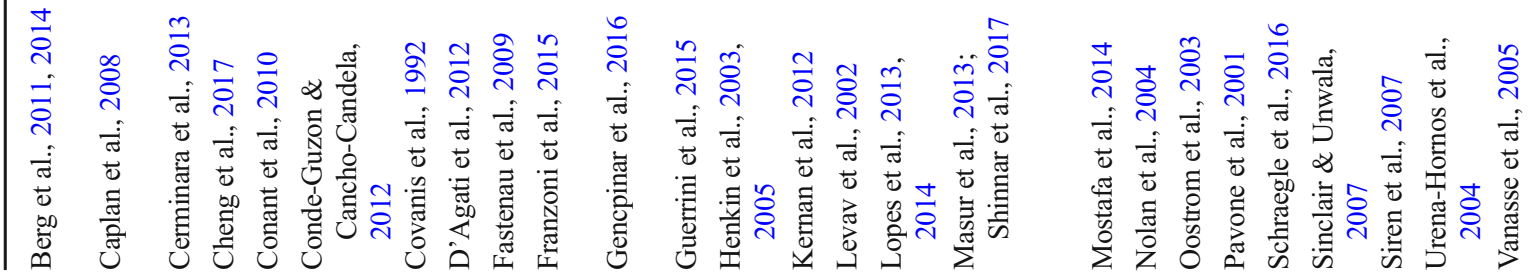


Children (WISC-III) was the psychological test most often used to assess general intelligence. Of 11 studies reporting on full-scale IQ, nine studies were suitable for pooling of results (Caplan et al., 2008; Conde-Guzon \& CanchoCandela, 2012; D'Agati et al., 2012; Gencpinar et al., 2016; Kernan et al., 2012; Lopes et al., 2013; Masur et al., 2013; Nolan et al., 2004; Pavone et al., 2001). The estimated mean full-scale IQ from the single-arm random-effects metaanalysis was 96.78 (95\% CI: 94.46-99.10; $T^{2}=7.57: I^{2}=$ $64.2 \%$ ) for absence patients, which is significantly different from the normative mean of 100 (Fig. 2). The mean full-scale IQ based on a sensitivity analysis excluding studies with a moderate risk of bias was 97.43 (95\% CI: 94.43 ; 100.43; $\left.T^{2}=10.84 ; I^{2}=75.9 \%\right)$. The estimated mean difference in IQ points compared to a control group in seven available case-control studies was -8.03 (95\% CI: $-10.45--5.61$; $T^{2}=0 ; I^{2}=0 \%$ ). The mean difference in IQ points in a sensitivity analysis excluding studies with a moderate risk of bias was -8.52 (95\% CI:-11.31 - -5.73; $T^{2}=0 ; I^{2}=0 \%$ ). The estimated mean full-scale IQ of the control group was 105.09 (95\% CI: $101.63-108.56 ; T^{2}=17.40 ; I^{2}=82 \%$ ), which is significantly higher than the normative mean of 100 .

Out of seven studies reporting on verbal IQ, four could be included in the meta-analysis with an estimated mean of 97.98 (95\% CI: 95.80-100.16; $T^{2}=0.28 ; I^{2}=5.5 \%$ ) and an estimated mean difference of -9.01 (95\% CI: $-12.11--5.90 ; T^{2}=$ $1.31 ; \mathrm{I}^{2}=12.85 \%$ ) compared to controls (Fig. 2).

Out of seven studies reporting on performance IQ, four were suitable for meta-analytic comparison with an estimated mean of 97.23 (95\% CI: 93.24-101.22; $T^{2}=15.86 ; I^{2}=$ $78.7 \%)$ and an estimated mean difference of $-5.32(95 \% \mathrm{CI}$ : $-8.27-2.36 ; T^{2}=0 ; I^{2}=0 \%$ ) compared to controls (Fig. 2).

The estimated mean for the Verbal Comprehension Index was 94.43 (95\% CI: 91.11-97.75; $T^{2}=7.39 ; I^{2}=70.2 \%$ ), the estimated mean for the Processing Speed Index was 96.30 (95\% CI: 93.74-98.86; $T^{2}=2.77 ; I^{2}=41.9 \%$ ) and the estimated mean for the Perceptual Organization Index was 97.09 (95\% CI: $95.50-98.69 ; T^{2}=0 ; I^{2}=0 \%$ ). One study, which used the WISC-IV, reported a mean Working Memory Index of 94.8 (SD 14.2). Sensitivity analyses by excluding outliers based on the funnel plots did not yield different conclusions. Results on the subtests of the Wechsler Intelligence tests are available in Table S1. Non-verbal intelligence tests were used in three studies. In the study with by far the largest sample of Masur et al. $(n=316)$ non-verbal intelligence assessed with the TONI-3 test was significantly higher compared to the normative mean (Masur et al., 2013). Raven's Progressive Matrices was used by Oostrom et al. which reported no significant difference from normative values in a small sample $(n=10)$ of children with either CAE or JAE (2003). A simplified version of the Raven's Progressive Matrices was used by Cheng et al. in children with CAE $(n=43)$ which performed significantly worse compared to controls (2017). 


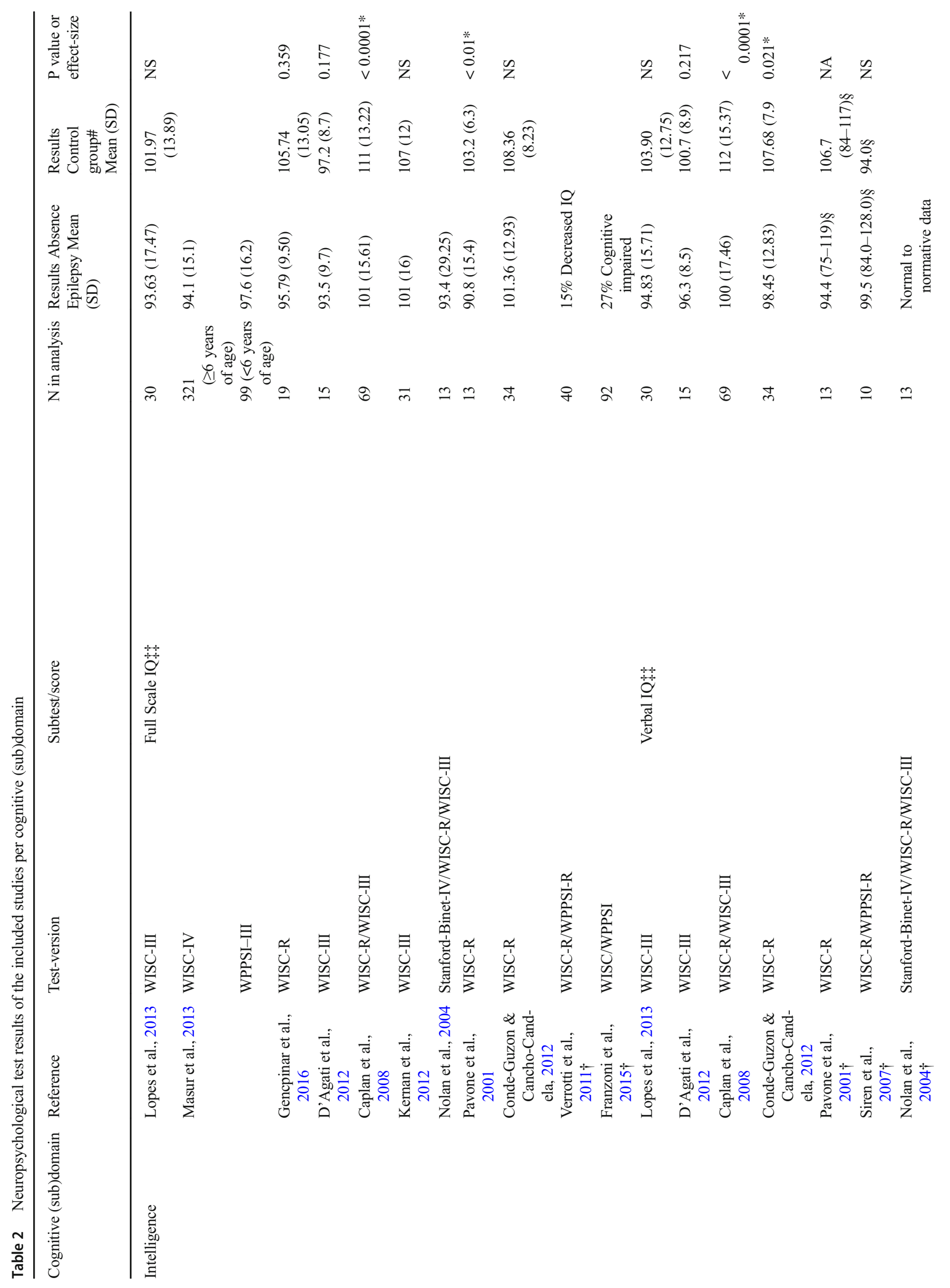




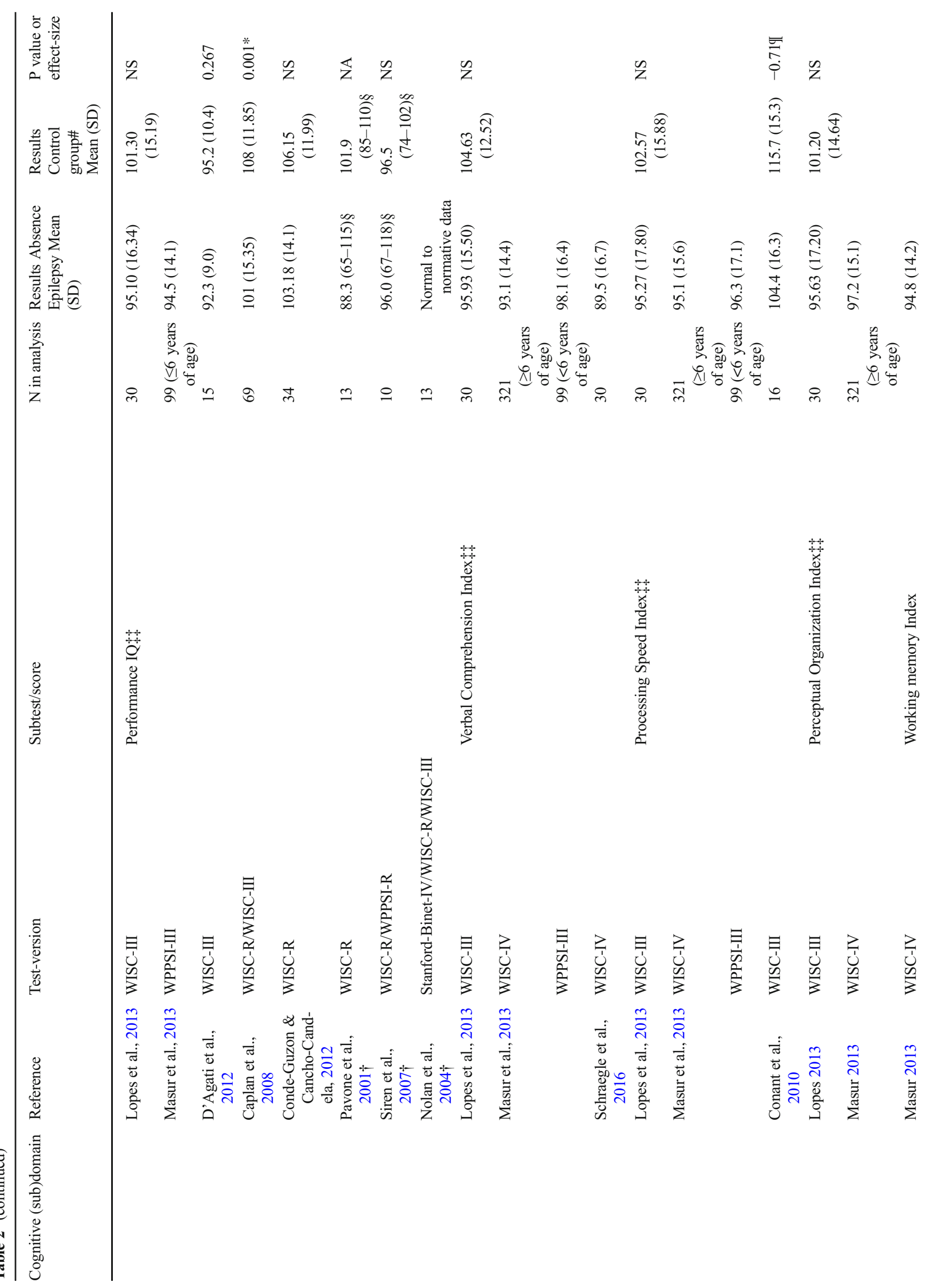




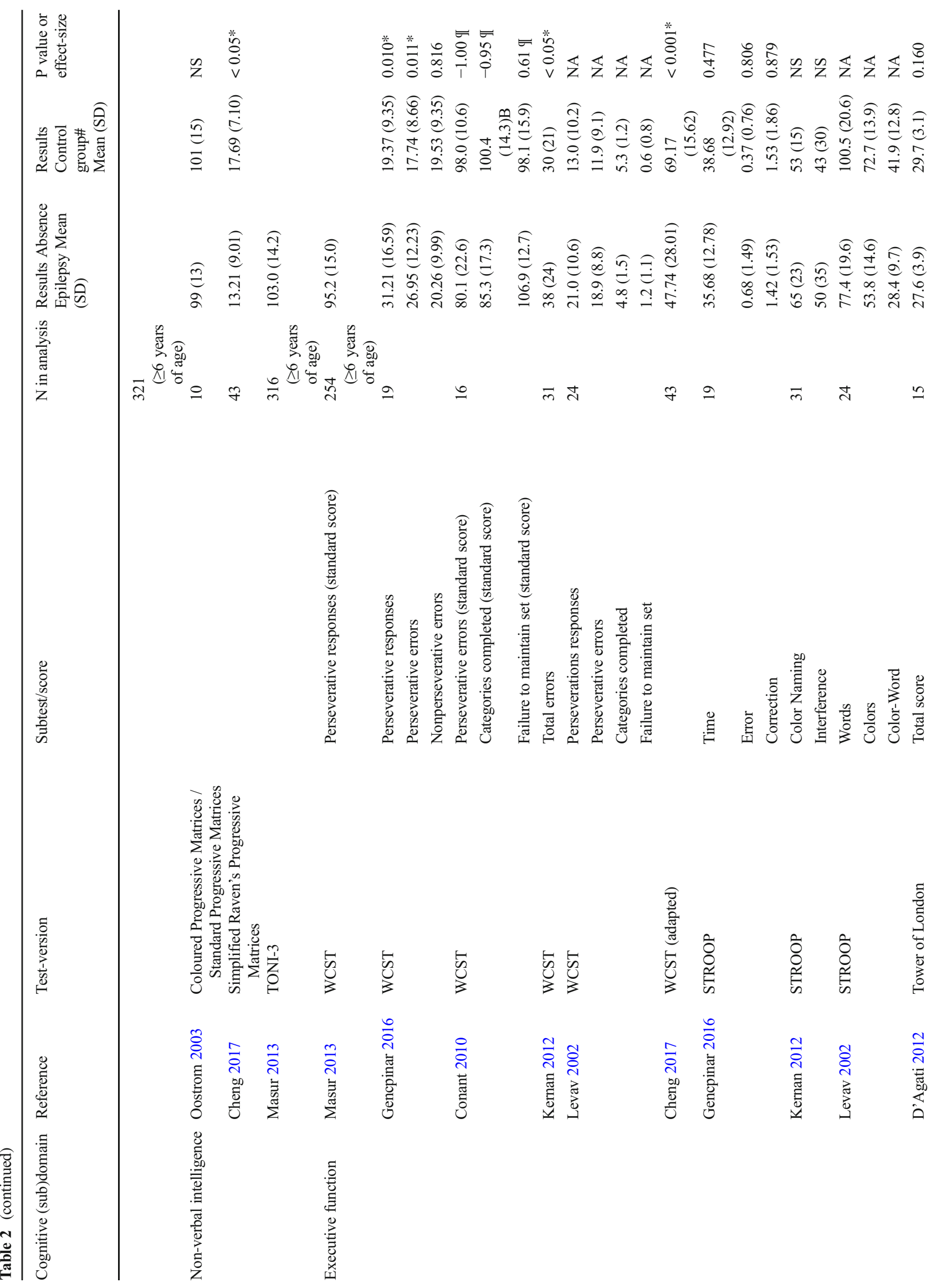




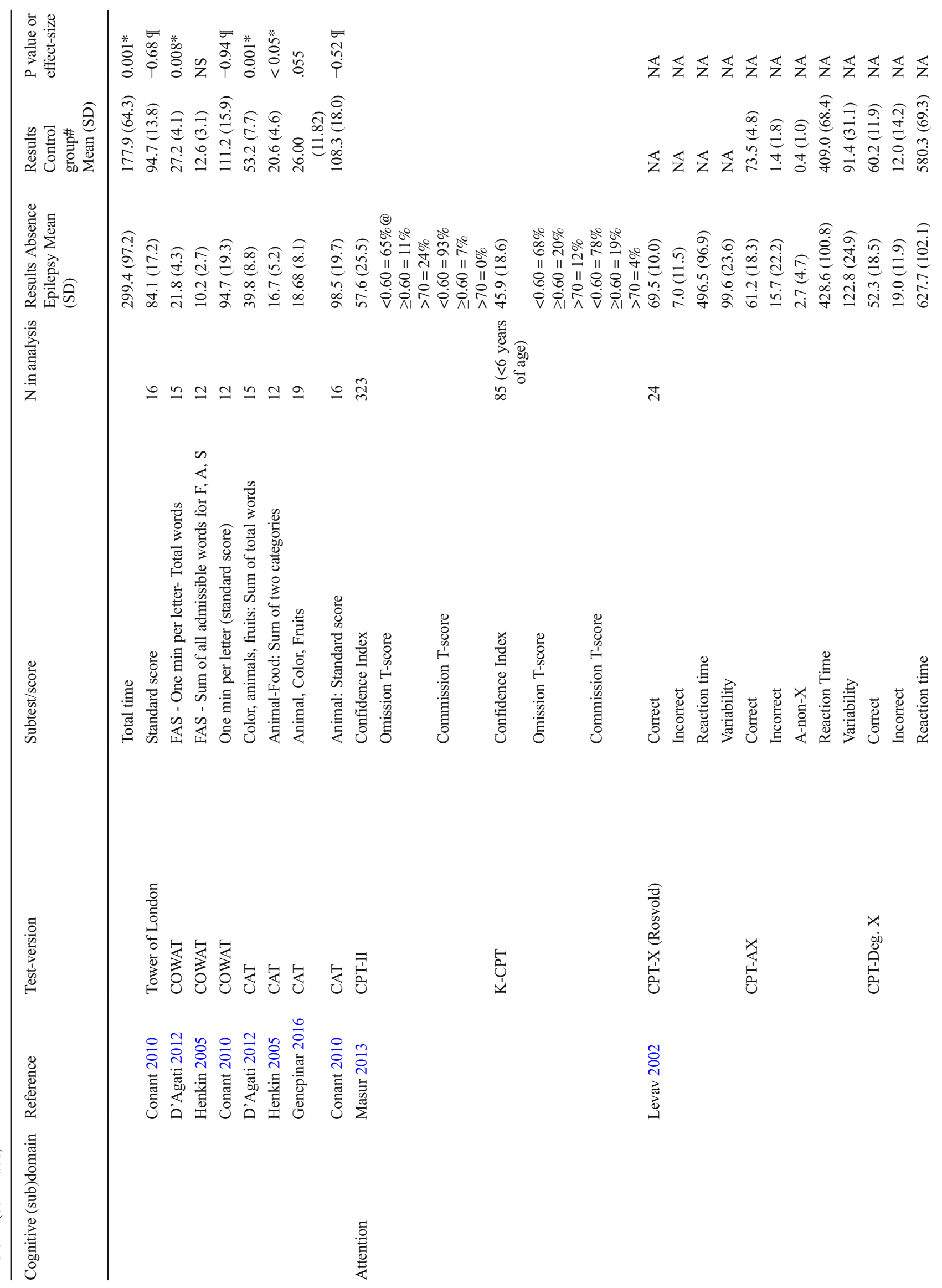




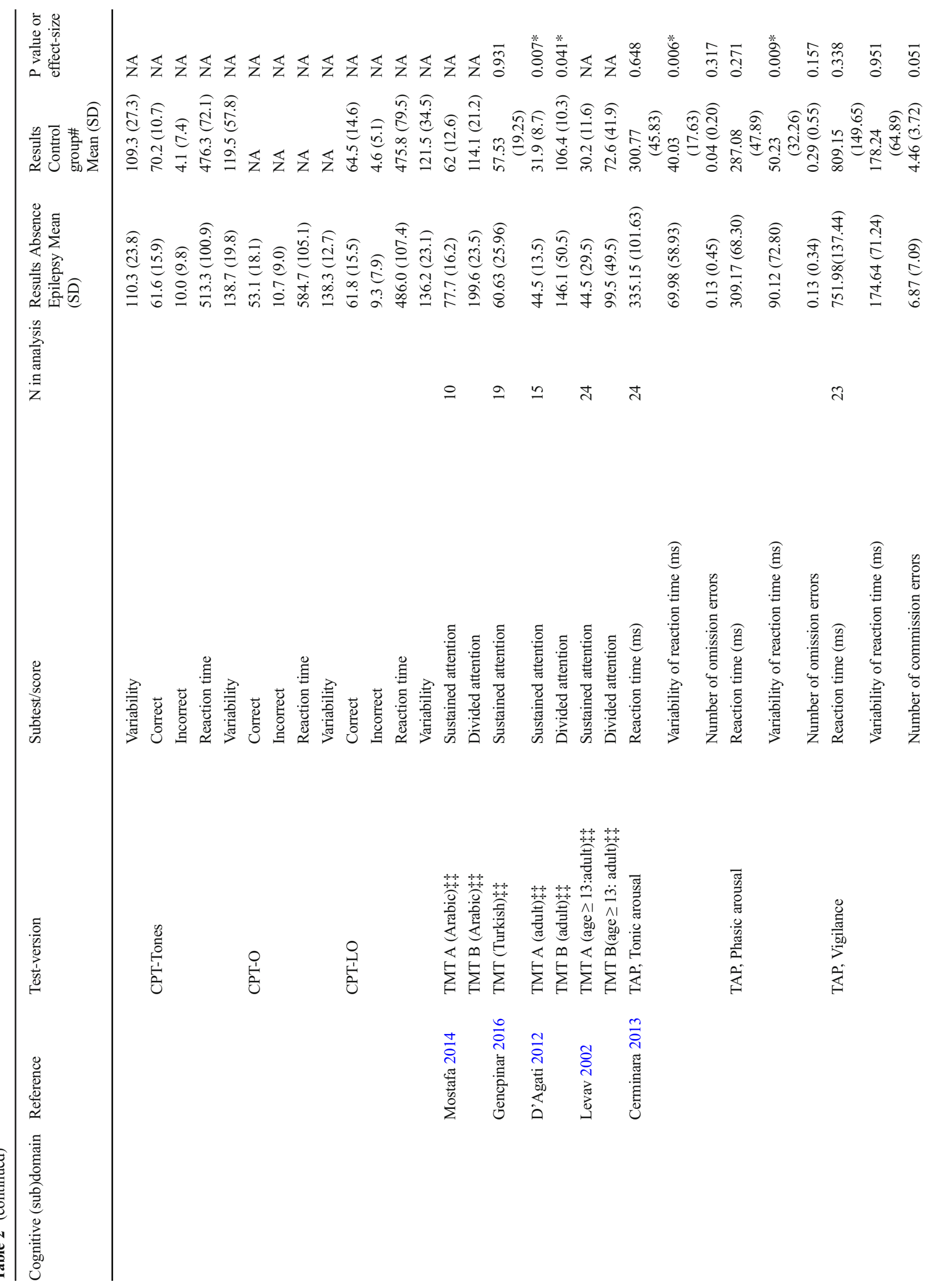




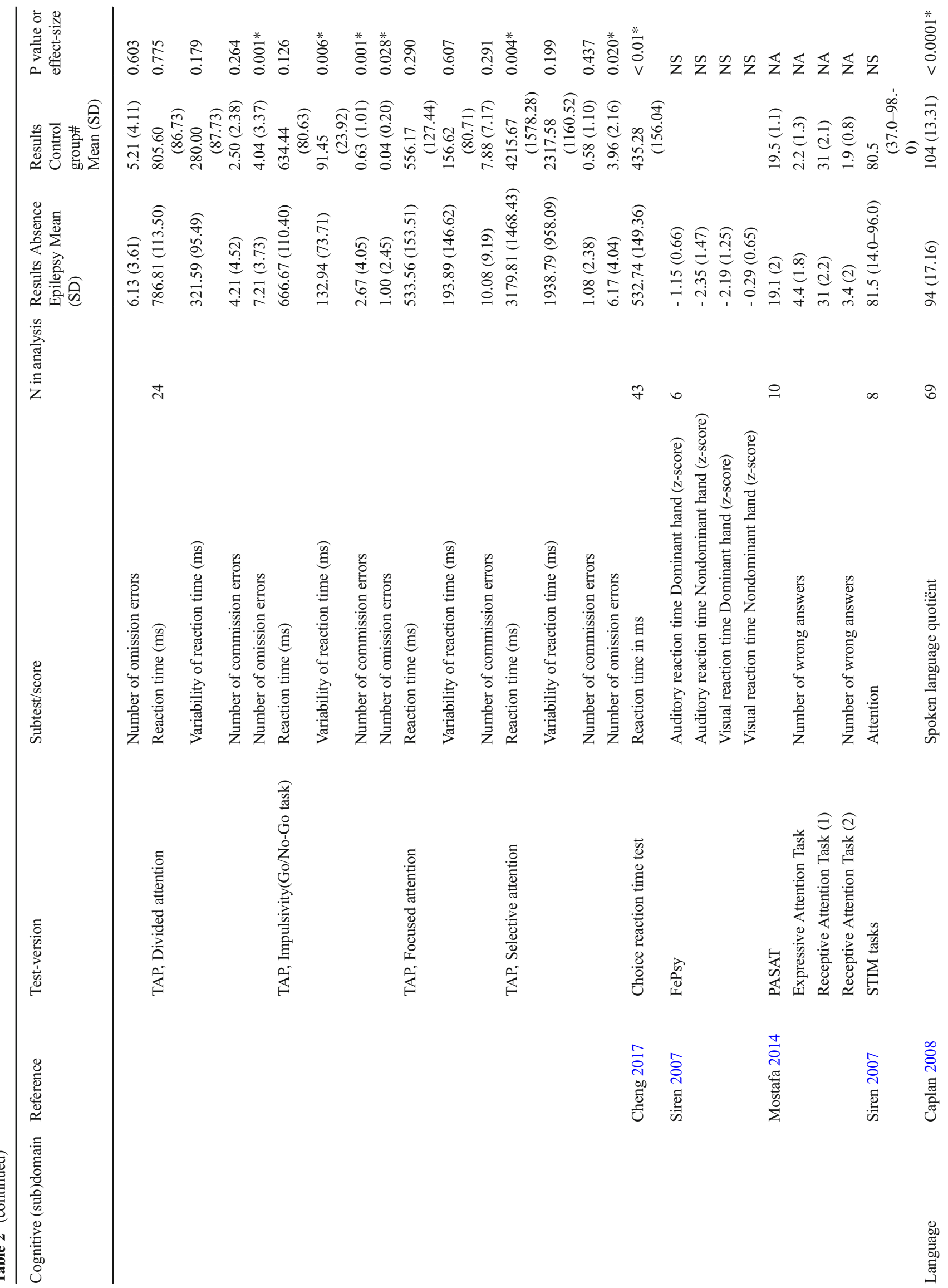




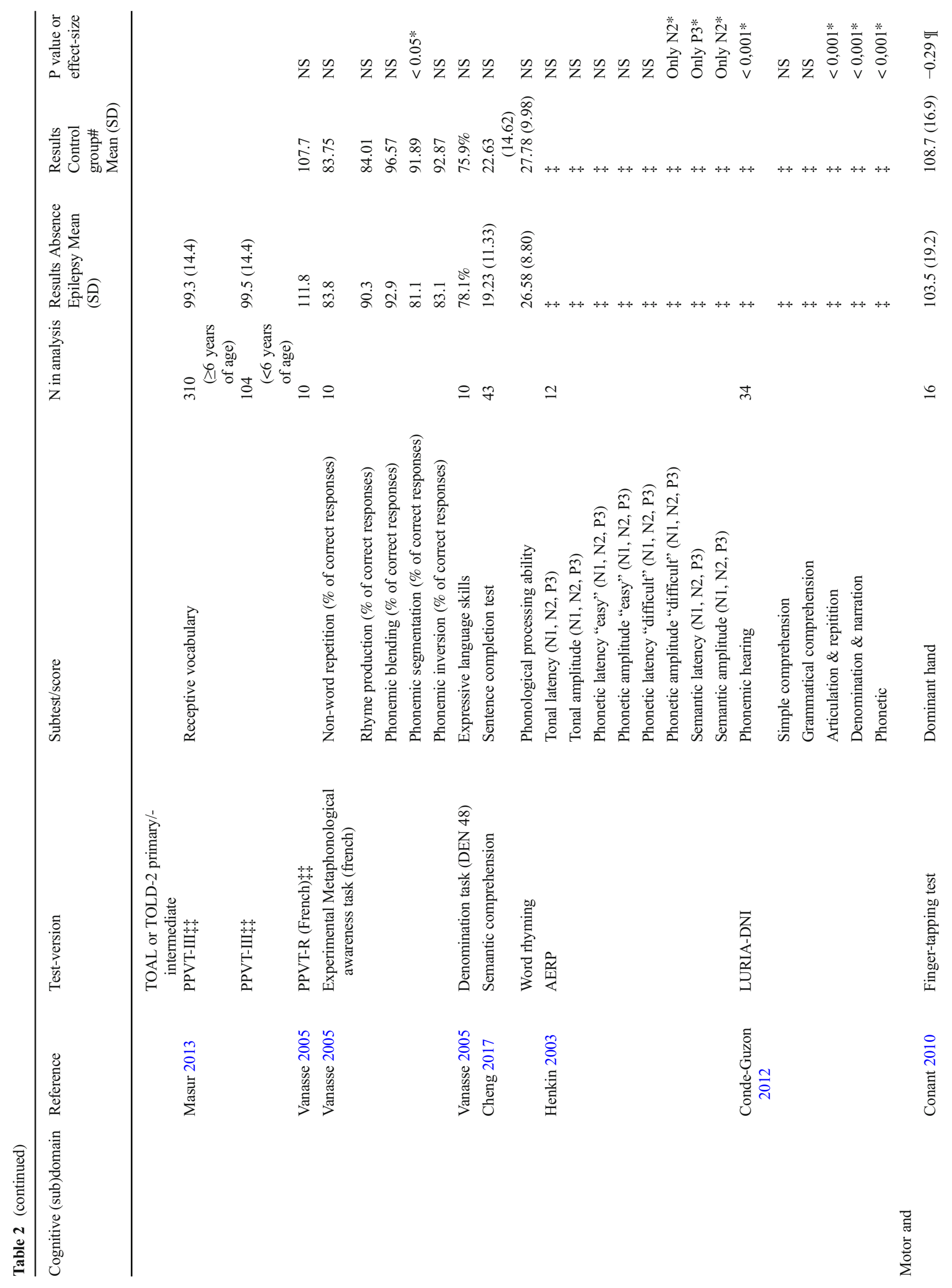




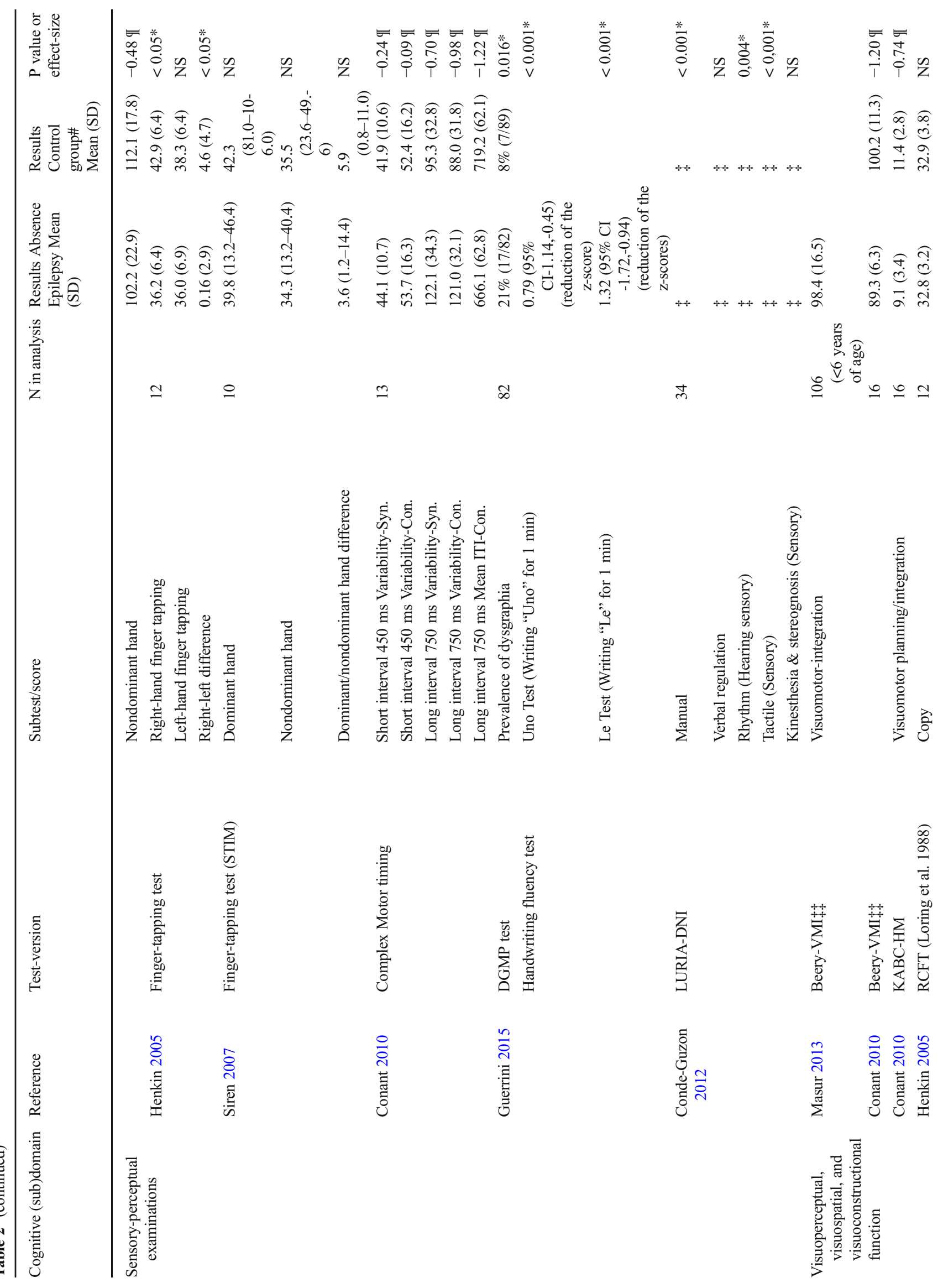




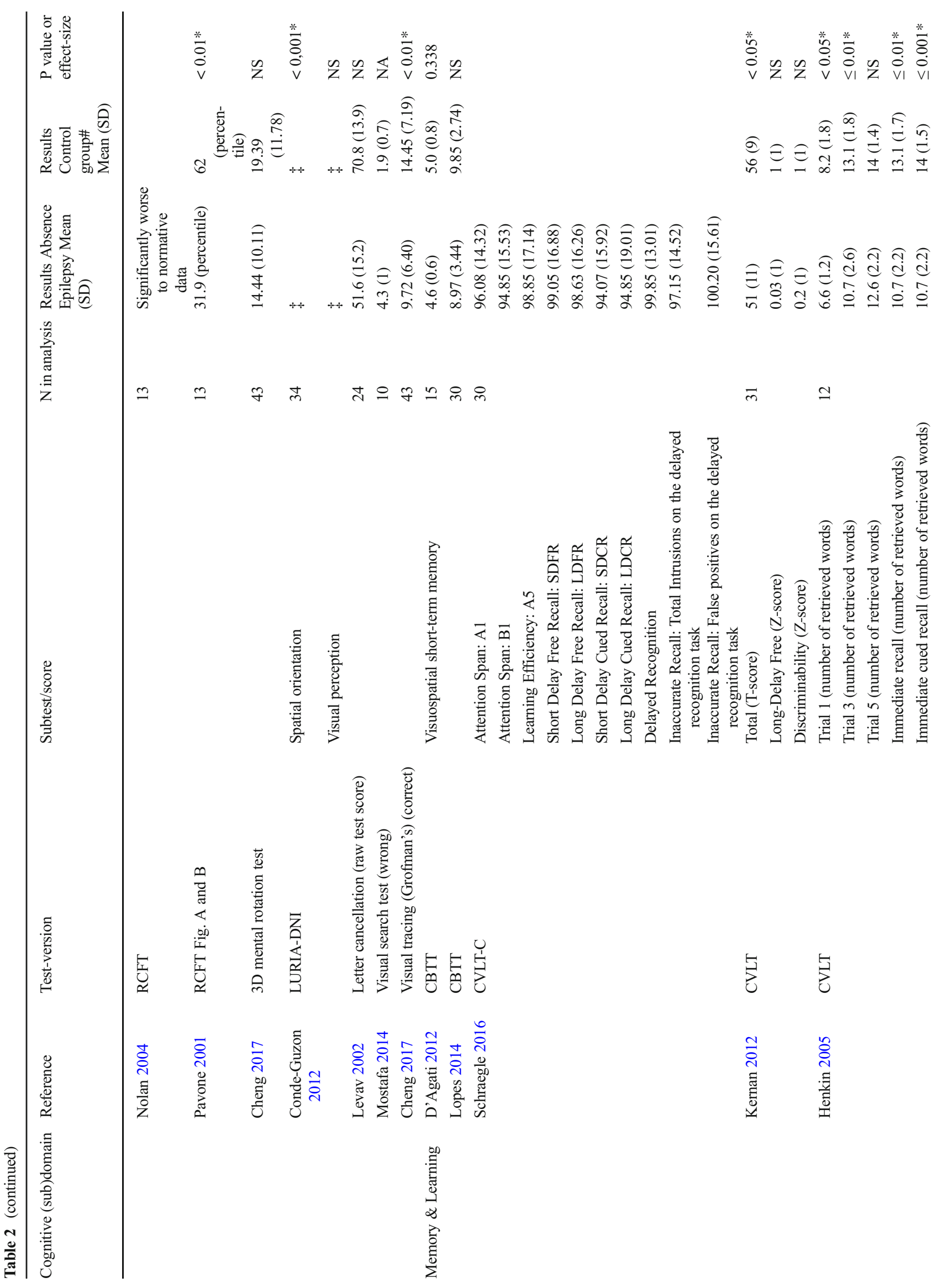




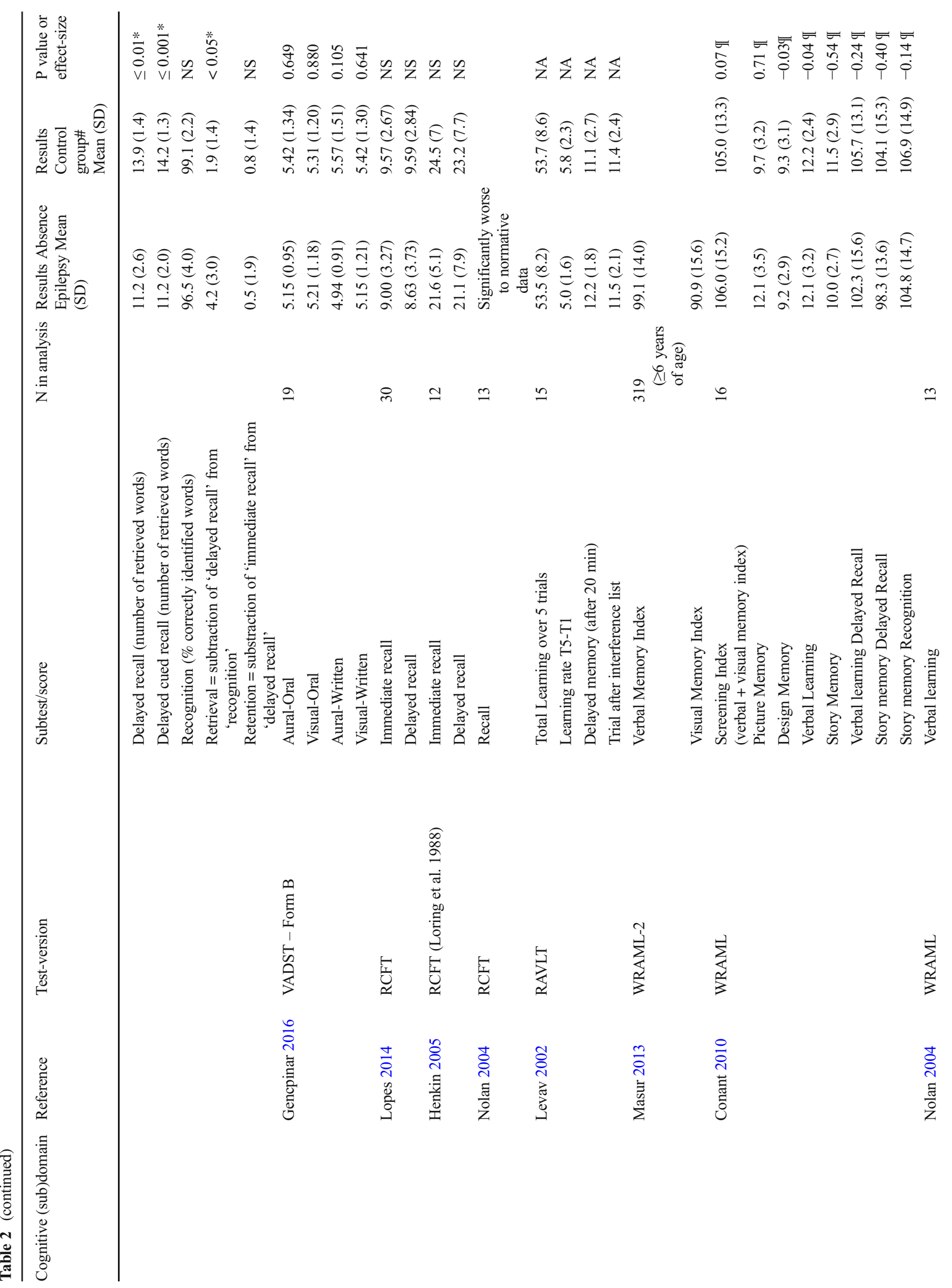




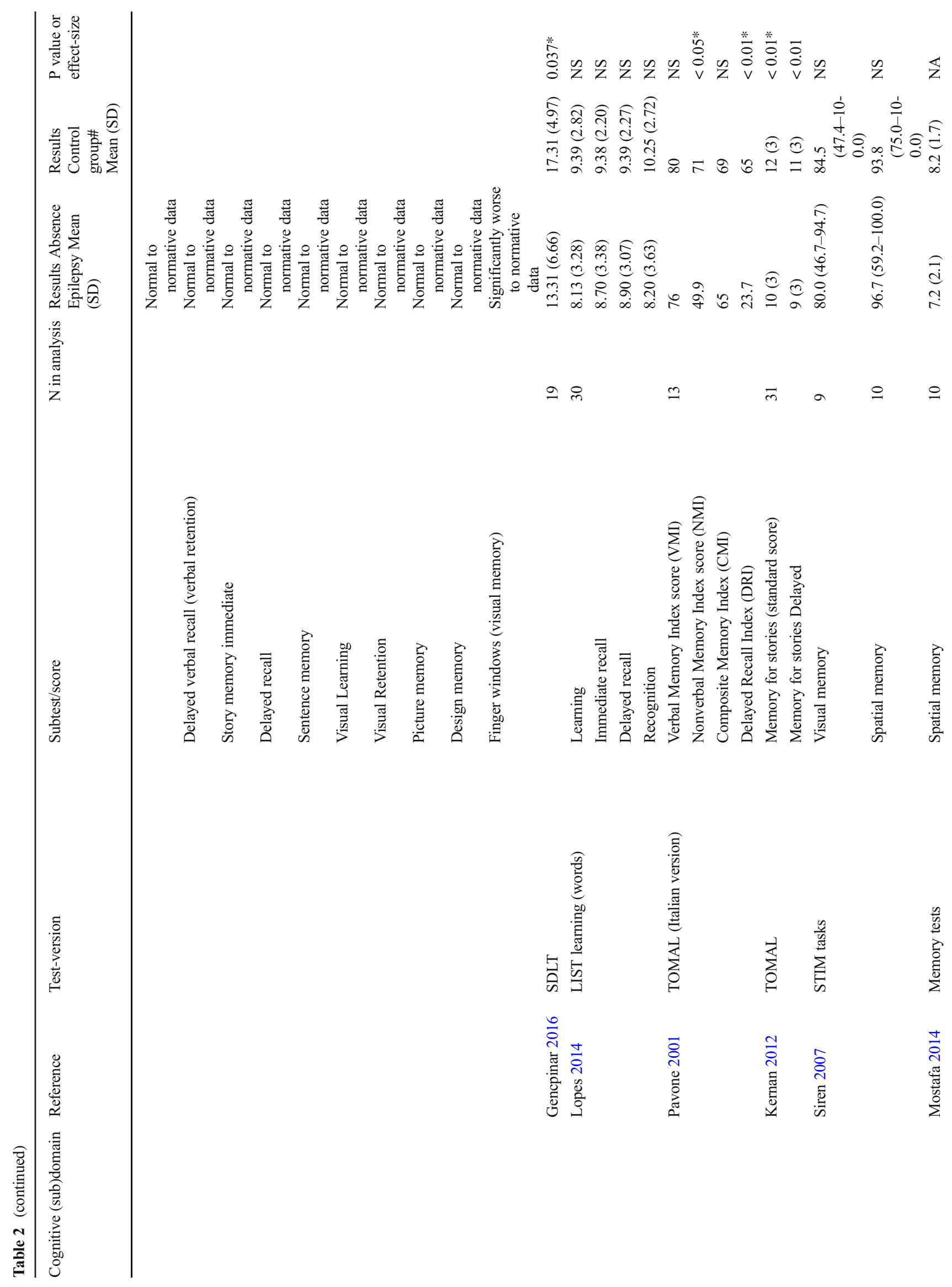




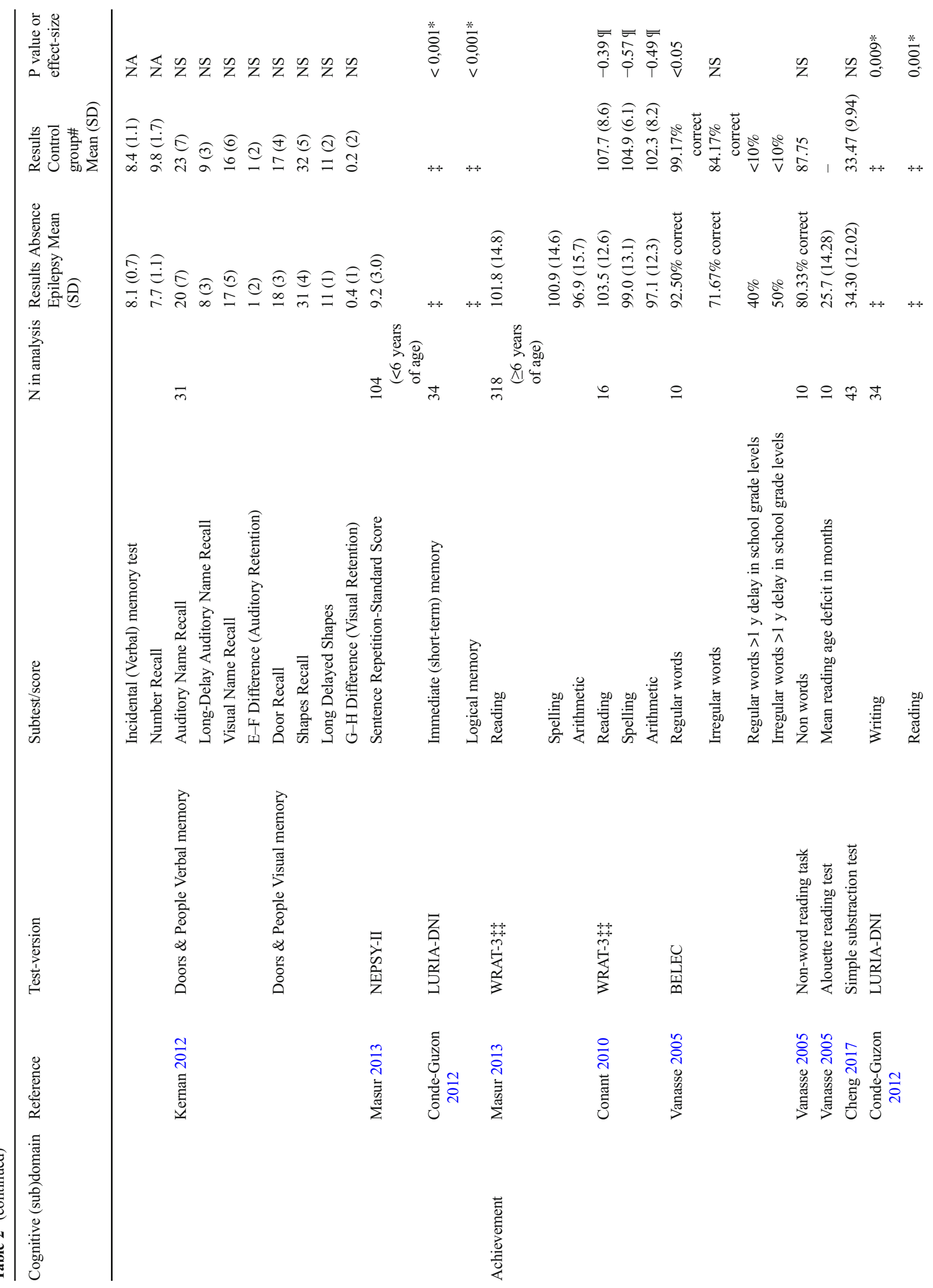




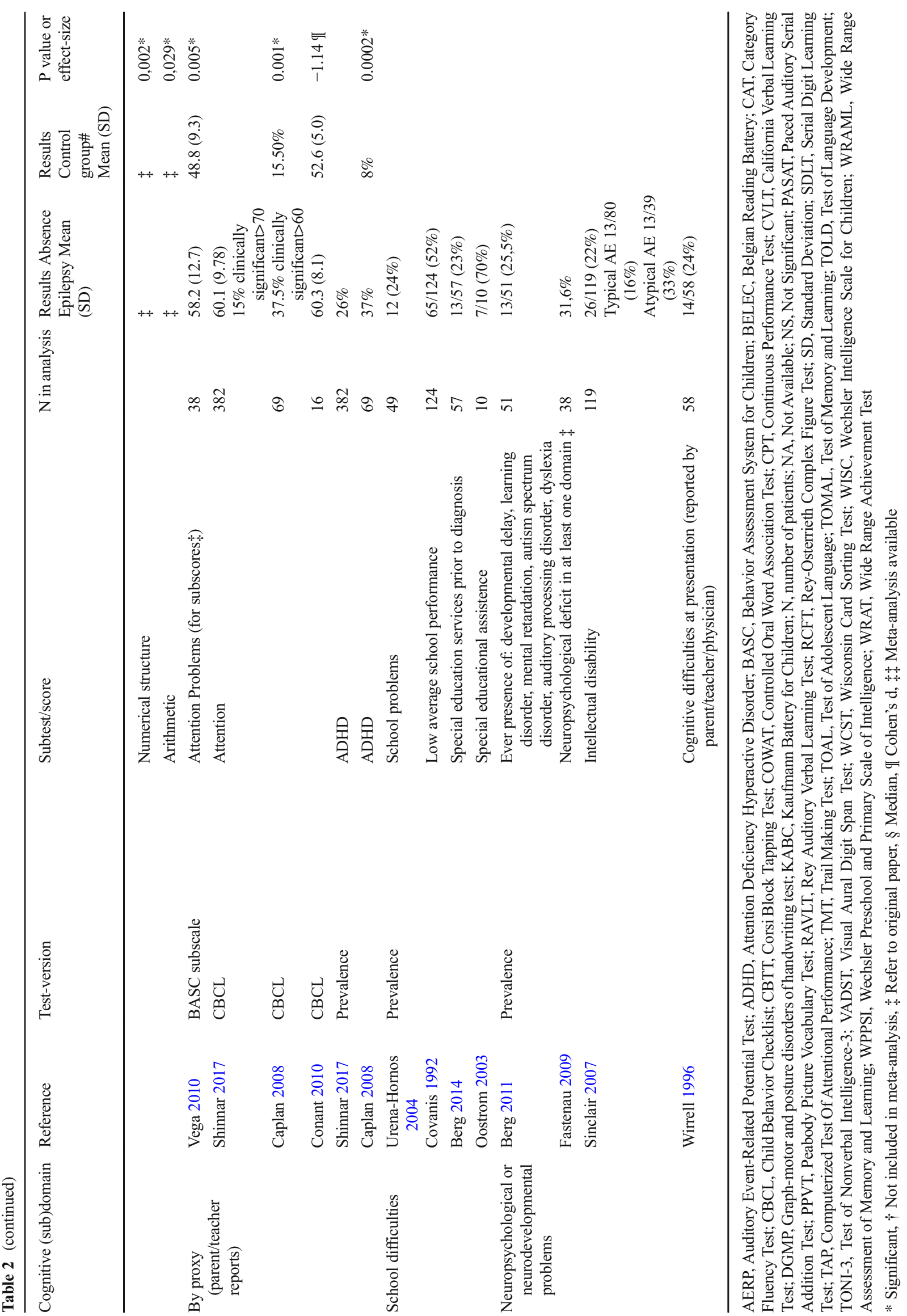




\section{Executive Function}

A total of eight studies assessed executive function (Cheng et al., 2017; Conant et al., 2010; D'Agati et al., 2012; Gencpinar et al., 2016; Henkin et al., 2005; Kernan et al., 2012; Levav et al., 2002; Masur et al., 2013) using different instruments. The performance of children with $\mathrm{AE}$ on the STROOP test did not significantly differ from controls (Gencpinar et al., 2016; Kernan et al., 2012; Levav et al., 2002). Significant worse performance was noted in multiple studies using the Wisconsin Card Sorting Test (Cheng et al., 2017; Conant et al., 2010; Gencpinar et al., 2016; Kernan et al., 2012; Levav et al., 2002; Masur et al., 2013), the Category Fluency Test (Conant et al., 2010; D'Agati et al., 2012; Henkin et al., 2005) and the Tower of London Test (Conant et al., 2010; D'Agati et al., 2012). In two out of three studies lower function was noted on verbal fluency with the Controlled Oral Word Association Test (Conant et al., 2010; D'Agati et al., 2012; Henkin et al., 2005). The control group in the study of Conant et al. scored higher than the normative mean; while the children with AE scored approximately average. Pooling of results was not possible for these tests due to differences in administration of the neuropsychological test and/or outcome reporting.

\section{Attention}

A total of eight studies assessed attention (Cerminara et al., 2013; Cheng et al., 2017; D'Agati et al., 2012; Gencpinar et al., 2016; Levav et al., 2002; Masur et al., 2013; Mostafa et al., 2014; Siren et al., 2007). A large randomized clinical trial in CAE showed impairments in attention in up to $1 / 3$ of patients with the Conner's Kiddie Continuous Performance Test in patients just started with anti-epileptic drug monotherapy $(<1$ week) or before starting treatment (Masur et al., 2013). Errors of omission (missing relevant targets) were more common than errors of commission (responding to non-targets). During follow-up, attentional deficits persisted independent of anti-epileptic drug treatment or seizure control. The study of Levav et al. used the Rosvold Continuous Performance test and also reported diminished scores in visual sustained attention (2002). However, in this study, the mean age of the control group used was 12 years older than the group with AE.

On the Trail Making Test A, the pooled estimated mean difference compared to controls was 12.37 s (95\% CI: 7.02$17.73 ; T^{2}=0 ; I^{2}=0 \%$ ) longer for children with $\mathrm{AE}$ and on the Trail Making Test B an estimated mean difference was found of 51.48 s (95\% CI: $\left.12.49-90.46 ; T^{2}=1061.59 ; I^{2}=89.9 \%\right)$ (Fig. 2) (Conant et al., 2010; D’Agati et al., 2012; Levav et al., 2002; Mostafa et al., 2014).

Furthermore, Cerminara et al. found significantly lower scores in some measures of alertness, divided attention, impulsivity, and selective attention in CAE compared to controls (2013). The divided and selective attention tasks were characterized by more errors of omission, whereas the impulsivity task was characterized by more commission errors. Reaction times had significantly more variability during the tonic arousal, phasic arousal and impulsivity task, but not in the divided or selective attention task. Focused attention did not differ from controls; however, Cheng et al. (2017) did find a significantly longer choice reaction time, which is similar to the focused attention task in the study performed by Cerminara et al. (2013).

Mostafa et al. reported significantly lower mean scores compared to controls in an expressive attention and receptive attention task; however, it was not clear whether this was still statistically significant when corrected for the 6 years age difference with controls (2014). Siren et al. used FEPSY auditory/visual reaction times and STIM tasks to assess attention and did not find a significant difference in this small sample $(n=10)(2007)$.

\section{Language}

A total of six studies assessed language (Caplan et al., 2008; Cheng et al., 2017; Conde-Guzon \& Cancho-Candela, 2012; Henkin et al., 2003; Masur et al., 2013; Vanasse et al., 2005). Caplan et al. reported significantly lower scores on the Spoken Language Quotiënt (SLQ) using the Test of Language Development (TOLD) compared to a control group (2008). Vanasse et al. used a metaphonological awareness task and reported significant worse phonemic segmentation compared to controls (2005). None of the other variables in this test differed from controls.

Masur et al. and Vanasse et al. did not find a significant difference in receptive vocabulary using the Peabody Picture Vocabulary Test (PPVT) compared to normative values (Masur et al., 2013; Vanasse et al., 2005). Vanasse et al. additionally used a denomination test to assess expressive language in a small sample of 10 subjects, but this did not yield a significant difference (2005).

The study of Conde-Guzon and Cancho-Candela in typical AE patients reported significantly lower performance in phonemic hearing, articulation/repetition, denominating/narration and phonetic analysis (2012). Comprehension and understanding of simple grammar did not differ significantly.

In a recent study by Cheng et al. semantic comprehension and word rhyming was not significantly worse compared to controls (2017). Furthermore, Henkin et al. reported on auditory event-related potentials and found significant increased N2 amplitudes for phonetic and semantic processing, as well as, a significantly increased latency for semantic stimuli for P3 compared to controls (2003). 

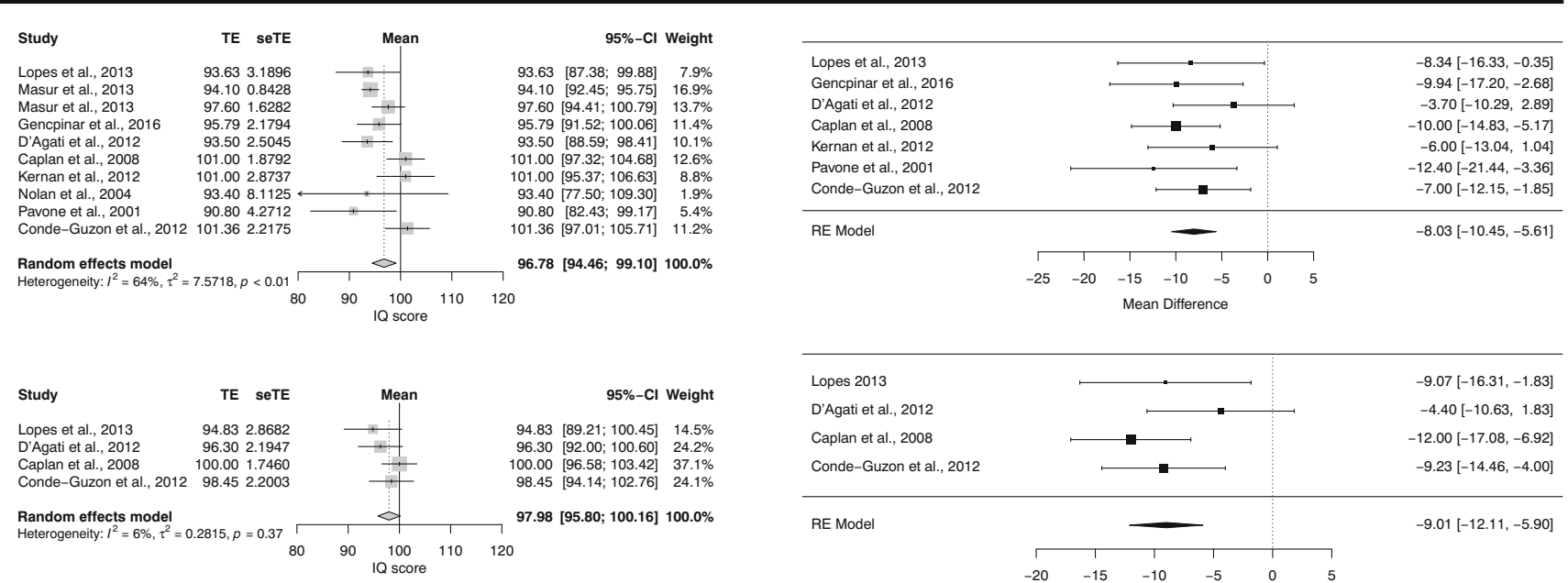

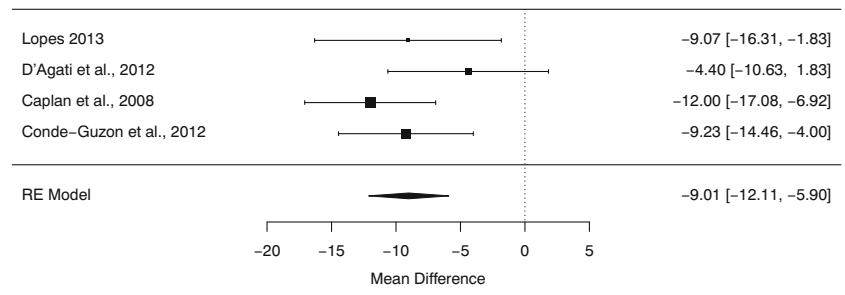

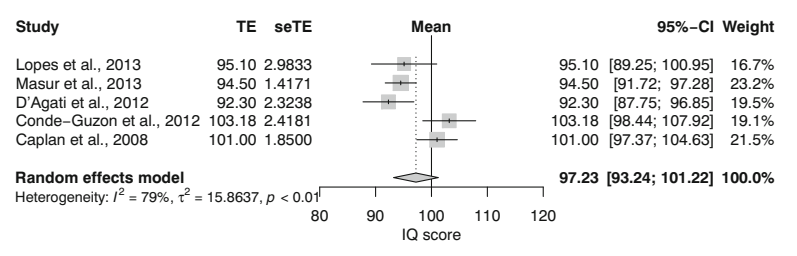

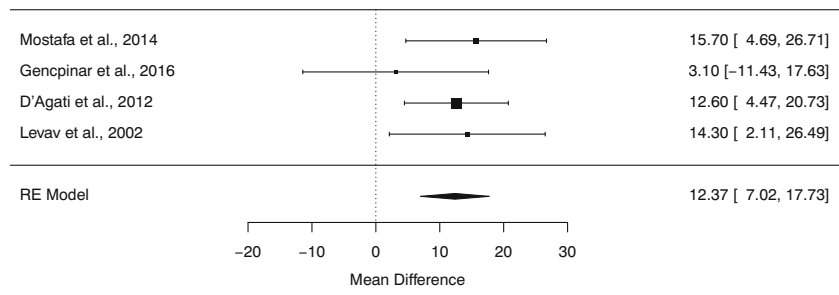
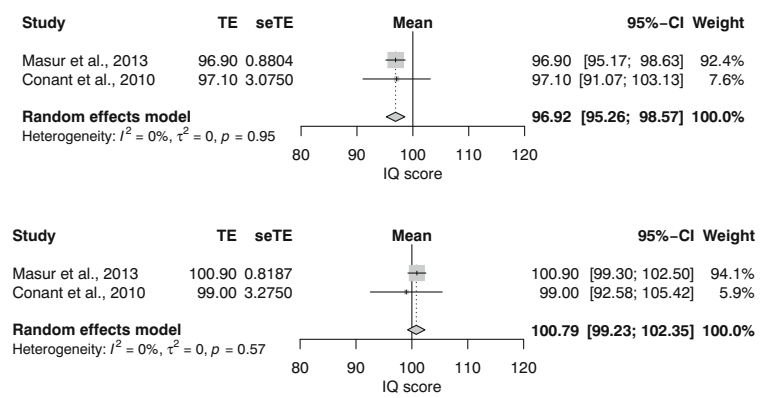

Fig. 2 Forest plots of the single-arm meta-analyses (weighted average) and meta-analyses of the mean difference (difference in performance in case-control studies). For each study the mean is represented by a square

\section{Motor and Sensory-Perceptual Examinations}

Five studies have investigated motor function (Conant et al., 2010; Conde-Guzon \& Cancho-Candela, 2012; Guerrini et al., 2015; Henkin et al., 2005; Siren et al., 2007). Siren et al. performed a finger-tapping test in the dominant and nondominant hand but did not find significant differences compared to controls (2007). Conant et al. also reported normal fine motor speed using a finger tapping test in the dominant

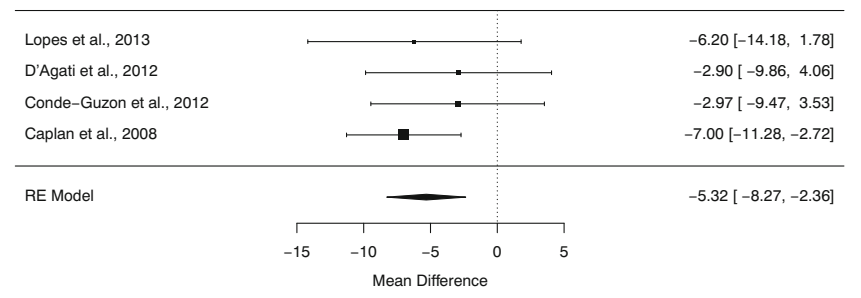

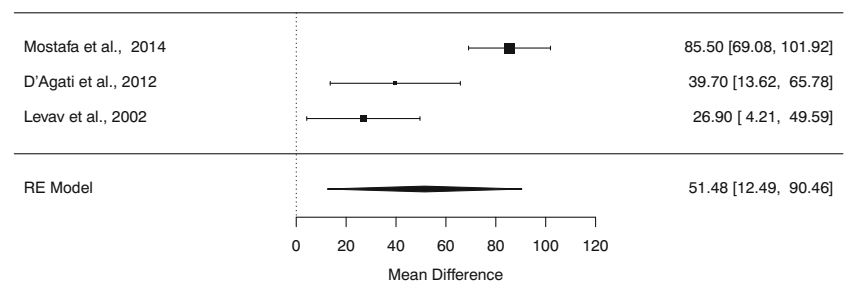
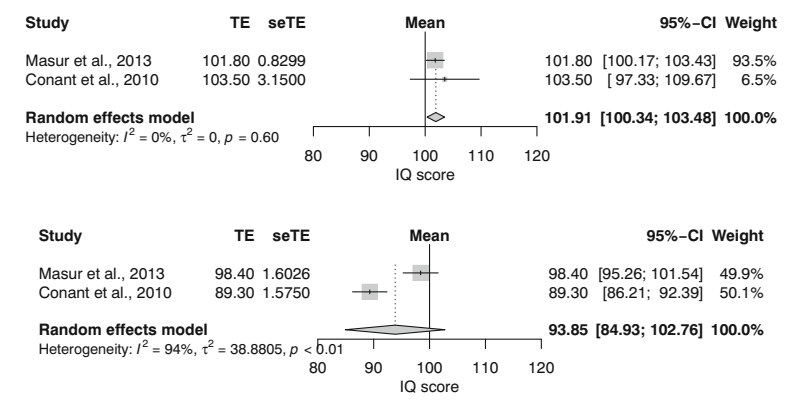

(size is proportional to the study's weight) and the $95 \%$ confidence inter$\mathrm{val}(\mathrm{CI})$ is represented by a horizontal line. The overall weighted mean is represented by a diamond shape

and non-dominant hand (2010). The control group in the study of Conant et al. scored higher than the normative mean; while children with AE scored approximately average. Conant et al. also assessed complex motor control and reported worse performance in AE compared to controls. Pooling of results with the finger tapping test was not possible due to differences in test protocols and reporting of results. Contrarily, Henkin et al. did find a significantly lower amount of taps per trial with finger-tapping in the right hand but not in the left-hand (2005). 
Furthermore, Guerrini et al. reported a higher prevalence of dysgraphia in AE (21\% vs. $8 \%$ for controls) and reported diminished overall performance in a handwriting fluency test compared to controls (2015). Conde-Guzon and CanchoCandela used the LURIA-battery to assess motor and sensory functions compared to control subjects (2012). They reported significantly lower performance in the manual subtest, but verbal regulation did not differ from normative values. Sensory functions such as rhythmic hearing and tactile subtests were significantly worse compared to controls, while kinesthesia and stereognosis did not differ from normative values.

\section{Visuoperceptual, Visuospatial, and Visuoconstructional Function}

Two studies assessed visuomotor planning and integration. Of the studies using the Beery-VMI, the largest study from Masur et al. $(n=106)$ reported a mean of 98.4 (SD 16.5), which is well within normal limits in children with CAE younger than 6 years of age (2013). Conant et al. included a smaller subset of patients $(n=16)$ and reported a significantly lower score of 89.3 (SD 6.3) compared to controls (2010). The single-arm random effect meta-analyses for the Beery-VMI was estimated at a mean of 93.85 (95\% CI: 84.93-102.76; $T^{2}=38.88$; $I^{2}=93.9 \%$ ), which is not significantly different from a normative mean of 100 (Fig. 2).

On the contrary, Conant et al. found significant lower performance using the KABC-HM (imitation of hand movements) a test for visuomotor planning and integration (Conant et al., 2010).

Five studies have investigated visuospatial skills. In total three studies used the Rey-Complex Test (RCFT). Nolan et al. found significant worse performance in the RCFT compared to normative data but did not include the average scores (2004). Pavone et al. found lower performance in the RCFT compared to the control group, although still within normal clinical range (2001). Henkin, et al. did not find a significant difference in the RCFT with the control group (2005). Cheng et al. (2017) reported that children with CAE did not perform worse in a $3 \mathrm{D}$ mental rotation test, however, Conde-Guzon and Cancho-Candela did find significant worse performance in the visuospatial subtest of the LURIA-DNI neuropsychological battery (2012).

Two studies assessed visual search ability. Levav et al. used a Letter Cancellation test and reported a large difference in completing the test compared to controls, however, the age difference between patients with CAE and controls was $\sim 12$ years (2002). In a study by Mostafa et al. total duration of a Visual Search Test was significantly worse compared to controls, however, controls were 6 years older on average (2014).

\section{Learning and Memory}

Fourteen studies assessed memory function (Conant et al., 2010; Conde-Guzon \& Cancho-Candela, 2012; D’Agati et al., 2012; Gencpinar et al., 2016; Henkin et al., 2005; Kernan et al., 2012; Levav et al., 2002; Lopes et al., 2014; Masur et al., 2013; Mostafa et al., 2014; Nolan et al., 2004; Pavone et al., 2001; Schraegle et al., 2016; Siren et al., 2007). Pavone et al. used the Test Of Memory And Learning (TOMAL) and found significant impairments in the Nonverbal Memory Index Score, Delayed Recall Index and Kernan et al. found significant differences in the Memory for Stories Subtest Scores compared to control subjects (Kernan et al., 2012; Pavone et al., 2001).

Schraegle et al. reported intact verbal memory and list learning compared to normative data using the California Verbal Learning Test (auditory memory) (2016). However, Kernan et al. (2012) and Henkin et al. (2005) did find significant memory impairment using this test compared to control subjects. Kernan et al. reported a significant difference in the total mean score, whereas Henkin et al. reported significant differences in immediate recall, delayed recall, and retrieval.

Findings on the recall of the Rey-Osterrieth Complex Figure Test (visual memory) did not differ compared to controls, although Nolan et al. reported significant worse performance compared to normative data (Henkin et al., 2005; Lopes et al., 2014; Nolan et al., 2004).

In the Randomized Clinical Trial of Masur et al. the Wide Range Assessment of Memory and Learning (WRAML) test showed a normal Verbal Memory Index, whereas the Visual Memory Index was abnormal (2013). In addition, Conant et al. observed the largest differences between children with AE and controls in picture memory and story memory subtests of the WRAML test (2010). Nolan et al. reported normal scores compared to normative data for all subtest of the WRAML test in a small study $(n=13)$ (2004). The Serial Digit Learning Test used by Gencpinar et al. revealed a significant difference compared to controls (Gencpinar et al., 2016). Number recall also seems diminished in the study of Mostafa et al., however controls were on average 6 years older (Mostafa et al., 2014).

Lopes et al. reported normal performance on LIST learning for words (2014). Masur et al. reported a Sentence Repetition mean standard score of 9.2 which is significantly lower compared to a normative mean of 10 in children with CAE younger than 6 years of age (2013). Conde-Guzon and CanchoCandela reported impairments in immediate (short-term) memory and logical memory in typical absence epilepsy compared to controls (2012). The performance on several other memory tests (Corsi Block Tapping Test (D'Agati et al., 2012; Lopes et al., 2014), Visual Aural Digit Span Test (Gencpinar et al., 2016), Rey Auditory Verbal Learning Test (Levav et al., 2002), Spatial Memory Test (Mostafa et al., 2014), Incidental 
Verbal Memory Test (Mostafa et al., 2014), STIM-tasks (Siren et al., 2007) and Doors and People (Kernan et al., 2012) did not differ in performance from control subjects.

\section{Achievement}

Five studies used an achievement test (Cheng et al., 2017; Conant et al., 2010; Conde-Guzon \& Cancho-Candela, 2012; Masur et al., 2013; Vanasse et al., 2005). Masur et al. (2013) and Conant et al. (2010) reported on arithmetic, reading and spelling ability using the Wide Range Achievement Test-3. On arithmetic, the estimated mean was 96.92 (95\% CI: 95.26-98.57; $T^{2}=0 ; I^{2}=0 \%$ ) (Fig. 2). On spelling, the estimated mean did not differ from normative data 100.79 (95\% CI: $\left.99.23-102.35 ; T^{2}=0 ; I^{2}=0 \%\right)$. However, the estimated mean on reading was 101.91 (95\% CI: 100.34-103.48; $T^{2}=$ $\left.0 ; I^{2}=0 \%\right)$. Vanasse et al. investigated reading ability in a small group $(n=10)$ and found lower scores across a regular, irregular and non-word reading task, albeit only the regular word reading task was significantly different from the control group (2005). The post-hoc administered Alouette reading task showed a mean reading deficit of 25.7 months (SD 14.28) in children with CAE.

In children with CAE, 40-50\% had more than 1-year delay in school grade levels compared to $10 \%$ of the control group. Conde-Guzon and Cancho-Candela found significant worse performance in writing, reading, numerical structure and arithmetic abilities compared to controls using the LURIA-DNI battery (2012).

\section{Reports on Attention or Attentional Deficiencies (by Proxy)}

Vega et al. reported attentional problems, especially forgetfulness and distractibility to be more prevalent in children with CAE compared to controls using the Behavior Assessment System for Children (2010).

The prevalence of attentional problems in $38 \%$ of children with $\mathrm{AE}$ differed significantly compared to $16 \%$ in control subjects assessed with the Child Behavior Checklist (CBCL) in the study by Caplan et al. (2008). However, Shinnar et al. (2017) reported a lower percentage of $15 \%$ clinically significant attentional problems in CAE compared to the study of Caplan et al. (2008) but used a higher cut-off value. Conant et al. (2010) and Shinnar et al. reported similar mean scores on attention, which in the study of Conant et al. was significantly higher than controls. Caplan et al. also reported a higher prevalence of $37 \%$ with a diagnosis of attentional deficit hyperactivity disorder (ADHD) versus $6 \%$ in control subjects in their population (2008). Shinnar et al. estimated a $26 \%$ prevalence of ADHD in drug-naïve children with CAE (2017).

\section{Prevalence of School Difficulties}

Four studies reported on school performance (Berg et al., 2014; Covanis et al., 1992; Oostrom et al., 2003; UrenaHornos et al., 2004). Urena-Hornos et al. reported school problems in 12 out of 49 (24\%) children with AE using a telephonic follow-up assessment (2004). Covanis et al. reported a low average school achievement in 65 out of 124 (52\%) children with CAE (1992). Berg et al. reported that 13 out of 57 (23\%) CAE patients had already received special education prior to diagnosis of epilepsy (2014). Oostrom et al. reported special educational assistance in 7 out of 10 children with either CAE or JAE (2003).

\section{Miscellaneous Data on Neurodevelopmental Problems}

Four studies assessed the prevalence of neuropsychological and/or neurodevelopmental problems (Berg et al., 2011; Fastenau et al., 2009; Sinclair \& Unwala, 2007; Wirrell et al., 1996). In the study of Berg et al., neurodevelopmental disorders (Table 2) were present in 13 out of 51 (26\%) patients (2014). Fastenau et al. reported that $32 \%$ of 38 patients had at least one neuropsychological deficit in at least one domain (see Table 2) (2009). Sinclair \& Unwala reported intellectual disability in $22 \%$ out of 119 children with CAE. Subgroup analysis showed intellectual disability in $16 \%$ of children with typical absences and $33 \%$ of children with AE and additional atypical features (2007). Wirrell et al. reported cognitive difficulties at presentation in $24 \%$ out of 58 children with $\mathrm{AE}$ (1996).

\section{Discussion}

The aim of this review was to systematically assess the literature on cognitive performance in AE. Children with $\mathrm{AE}$ are regarded to have cognitive functioning within normal range (Adie, 1924; Currier et al., 1963). Nevertheless, we found multiple studies reporting lower cognitive performance across a wide spread of cognitive domains. However, the exact degree of impaired cognitive functioning is difficult to estimate as the methodologies across studies vary and multiple neuropsychological tests have been used, which hampers comparisons between studies. Moreover, it is currently difficult to distinguish momentary effects on cognitive performance during the active stage of AE from long-lasting effects on cognitive functioning, as most studies reported on cognitive performance at different time-points after seizure onset. Some studies tested prior to introduction of anti-epileptic drug treatment, while others reported on a mixed population of 
children on or off anti-epileptic drug treatment and with or without ongoing seizures.

\section{Intelligence}

Full-scale IQ was estimated to be approximately three points lower on average compared to normative values. It is important to note that, although average performance in intelligence measures are statistically lower, the pooled averages still fall well within normal values. However, in case-control studies, the mean difference is larger with a difference of $\sim 8$ points in full-scale IQ, $\sim 9$ points in verbal IQ and $\sim 5$ points in performance IQ. There may be several explanations for these results. The mean IQ of the control subjects was significantly higher than normative values, which may simply resemble a higher average IQ in the studied population or geographical area. However, it may be due to exclusion of patients with a low IQ in several case-control studies (Caplan et al., 2008; D’Agati et al., 2012; Gencpinar et al., 2016; Henkin et al., 2005; Kernan et al., 2012; Lopes et al., 2013). Another explanation for a higher IQ in controls may be bias due to convenience sampling (e.g. children from academics), although this was not evident based on the methods used for the recruitment of control subjects in these studies. However, the estimated mean IQ in the single-arm meta-analysis in patients with AE is less subject to bias due to sampling error as a far larger proportion of the total population is being tested. Moreover, the estimated true variance $\left(T^{2}\right)$ for the single-arm meta-analyses was totally dependent on the three studies (Caplan et al., 2008; Conde-Guzon \& Cancho-Candela, 2012; Kernan et al., 2012) with the highest mean IQ scores in AE and controls. The estimated true variance $\left(T^{2}\right)$ was small for the pooled difference in case-control studies.

\section{Executive Function}

Lower than average performance in executive functioning was noted in cognitive flexibility, planning and verbal fluency (Cheng et al., 2017; Conant et al., 2010; D’Agati et al., 2012; Gencpinar et al., 2016; Henkin et al., 2005; Kernan et al., 2012; Levav et al., 2002; Masur et al., 2013). However, not all tests were indicative of lower executive functioning as results on the STROOP test did not differ.

\section{Attention}

There are clear indications for a lower performance in attention, such as sustained attention, selective attention and divided attention. From our meta-analysis, we can conclude that trail making $\mathrm{A}$ and $\mathrm{B}$ scores take significantly more time to perform by children with AE compared to controls, especially when attentional shifts (divided attention) are necessary. The estimated true variance $\left(T^{2}\right)$ for the trail making test B was high, however, this may be explained by differences in study design, as the study by Levav et al. reported in children $\geq 13$ years of age, which would probably require less seconds to finish the test than younger counterparts in the other two studies. Furthermore, in the study by Masur et al. sustained attention was mostly affected due to attentional lapses (errors of omission) rather than reflecting disinhibition (errors of commission) (2013).

\section{Language}

Results on specific language tests are of particular interest, as verbal IQ was estimated to be lower in case-control studies, and a relatively low mean verbal comprehension index (in children $>6$ years of age) was reported in the study by Masur et al. (2013). However, receptive vocabulary was not affected with the Peabody Picture Vocabulary Test in the study by Masur et al. (Masur et al., 2013). Two studies with a decent sample size raise concerns regarding expressive language as found by Caplan et al. (although average functioning is still within normal clinical range) and Conde-Guzon \& CanchoCandela (Caplan et al., 2008; Conde-Guzon \& CanchoCandela, 2012). However, these two studies were characterized by relatively high verbal IQ's in controls. Therefore, data on language tests in AE remains inconclusive, but warrants further research.

\section{Motor Function}

Studies on simple motor tasks were inconclusive (Conant et al., 2010; Henkin et al., 2005; Siren et al., 2007). Complex motor tasks may be impaired but were only assessed in one study (Conant et al., 2010) and the control group was characterized by higher scores than the normative mean. Furthermore, one study found a higher prevalence of dysgraphia and diminished performance in handwriting fluency. These findings were associated with abnormal neurophysiological findings, which led the authors to conclude that these patients had a form of dystonic dysgraphia. Interestingly, 12 out of 17 patients with initial dysgraphia were re-tested 5-years later and showed resolution of dysgraphia and improved handwriting skills. In addition, Conde-Guzon and Cancho-Candela also reported worse performance in a writing test (2012).

\section{Visuoperceptual, Visuospatial, and Visuoconstructional Function}

Visual-motor integration from the Beery-VMI test did not differ from the normative mean in our meta-analysis, although only two studies were available. The lower mean score in the Beery-VMI in the study by Conant et al. may be due to a small study effect. Studies on visual search tests and visuospatial skills remain inconclusive (Cheng et al., 2017; CondeGuzon \& Cancho-Candela, 2012; Henkin et al., 2005; Levav 
et al., 2002; Mostafa et al., 2014; Nolan et al., 2004; Pavone et al., 2001). Indeed, visual-spatial thinking ability in the meta-analysis of Loughman et al. in idiopathic generalized epilepsies did also not differ significantly in case-control studies (2014).

\section{Learning and Memory}

Results on memory have yielded inconclusive results. The largest study on memory by Masur et al. suggests lower visual memory function, although still within normal clinical range (2013). However, other studies on non-verbal memory tests have overall found average performance (Conant et al., 2010; D'Agati et al., 2012; Gencpinar et al., 2016; Henkin et al., 2005; Kernan et al., 2012; Lopes et al., 2014; Nolan et al., 2004; Pavone et al., 2001; Siren et al., 2007). Furthermore, studies on verbal memory mostly showed normal performance (Levav et al., 2002; Lopes et al., 2014; Schraegle et al., 2016). Nevertheless, some studies using subtests for memory for stories and with the California Verbal Learning Test did find lower performance compared to controls (Conant et al., 2010; Henkin et al., 2005; Kernan et al., 2012). However, the observed differences with controls in these studies might suggest a sample bias related to a higher functional level of the controls. Only, one study found an overall lower than average performance in memory functioning, especially in short-term auditory, visual memory and logical memory, although this study was also potentially characterized by a better than average control group as reflected by the performance of the control group on the WISC-R (Conde-Guzon \& Cancho-Candela, 2012). Sentence repetition was lower compared to the normative mean in the study by Masur et al. (2013), although scores still fall within normal clinical range and may be related to attentional deficits.

\section{Achievement}

Arithmetic ability may be vulnerable based on our metaanalysis of the WRAT-3 test, although test scores fall within the normal clinical range. Nevertheless, arithmetic ability was also worse compared to controls in children with $\mathrm{AE}$ in the study by Conde-Guzon and Cancho-Candela (2012). Evidence regarding reading ability, is contradictory, as the meta-analysis on the WRAT-3 test was not significantly lower, but other reading tests by Vanasse et al. (2005) and CondeGuzon \& Cancho-Candela (2012) did report worse performance compared to controls.

\section{By Proxy (Parent-Reported Functioning)}

Studies using parental questionnaires also point towards attentional problems and a higher prevalence of attentional deficit hyperactivity disorder.

\section{Prevalence of School Difficulties}

School difficulties seem more prevalent in patients with $\mathrm{AE}$ than in the normal population, as the three largest studies reported school difficulties in $23 \%$ to $52 \%$ (Berg et al., 2014; Covanis et al., 1992; Urena-Hornos et al., 2004).

\section{Prevalence of Neuropsychological and/or Neurodevelopmental Problems}

A prevalence of neuropsychological and/or neurodevelopmental problems were found in approximately $22 \%$ to $32 \%$ of the patients with CAE (Berg et al., 2014; Fastenau et al., 2009; Sinclair \& Unwala, 2007; Wirrell et al., 1996).

\section{General Discussion}

Cognitive deficits in one area may be related to the performance in another cognitive domain. Masur et al. reported a direct sequential effect among attention, memory, executive function, and academic achievement (2013). Therefore, the emergent pattern of clear attentional deficits in a proportion of children with absence epilepsy may influence performance in other cognitive domains. Nevertheless, the overall pattern suggests vulnerabilities in intelligence, attention and executive function. Less conclusive results were found for (expressive) language, motor function, visuo-perceptual functioning and learning \& memory. Ultimately, vulnerabilities in cognitive domains may impact neurocognitive development and lead to more academic difficulties.

How children with AE mature into adulthood is far less researched. The few studies that have investigated cognitive function during adulthood in patients with AE suggest that lower performance on neuropsychological tests may persist, but current studies are small in sample size. A study in $(n=10)$ adults with CAE in remission reported a full-scale IQ of 92 (69-99), a performance IQ of 85 (66-117), with particularly low scores in the picture arrangement, block design and object assembly subtests of the WAIS-R (Hommet et al., 2001). Language and executive function were also tested using specific tests, but did not differ with a control group. Recently Loughman et al. reported on cognitive function using the Woodcock-Johnson-III test of cognitive abilities in an adult population of genetic generalized epilepsy syndromes, including CAE $(n=10)$ and JAE $(n=21)$ patients $(2017)$. This study reported lower scores on brief intellectual ability, crystallized intelligence, new learning/memory and speed of processing (Loughman, Bowden, \& D'Souza, 2017). In conclusion, neurodevelopment may ultimately differ in AE, as a study also found abnormal cortical thickness connectivity in various regions after in young adults with a history of CAE (Curwood et al., 2015). 
What the impact may be on their working careers and psychosocial well-being is another important question. In a telephone follow-up study, $25 \%$ of 52 retrospectively identified patients of 11 to 36 years old with CAE had a history of psycho-pedagogical help (Martinez-Ferrandez et al., 2017). Another study in patients focusing on long-term psychosocial outcome in juvenile myoclonic epilepsy (JME) with a mean age over 60 years used AE patients as an epilepsy matched-control group (Holtkamp, Senf, Kirschbaum, \& Janz, 2014). To the surprise of the authors, a significantly lower amount of patients with a history of AE accomplished a university degree compared to controls. Nevertheless, the overall psychosocial outcome of this study was favorable. However, another study compared a group of patients with rheumatoid arthritis to patients with CAE and similar impact of "chronic disease" (Camfield \& Camfield, 2007). Young adults with a history of CAE were more prone to working in jobs requiring minimum education, having more behavioral problems and psychiatric consultations. Therefore, the potential impact later on in life cannot be underestimated and deserves further scientific attention.

Cognitive findings may also be related to (inter)ictal activity, pharmacological effects or another underlying (genetic) vulnerability, but disentangling all factors may not be feasible. The use of valproate was associated with attentional dysfunction in the study by Masur et al. (Cnaan et al., 2017; Dlugos et al., 2013; Glauser et al., 2010; Masur et al., 2013). In addition, seizure duration of more than 20 s correlated well with attentional dysfunction and errors of omission (Dlugos et al., 2013). In the prospective cohort study of Caplan et al. verbal IQ was associated with duration of illness and AED treatment. Moreover, ADHD was associated with the duration of illness and seizure frequency (2008). However, these associations have not been consistent across the literature.

Insights into possible underlying mechanisms are starting to be unraveled in recent years. Multiple studies using advanced magnetic resonance imaging (MRI) techniques in AE have for example found differences in brain regions or networks which are associated with executive function (Bai et al., 2011; Berman et al., 2010; Caplan et al., 2009; Carney, Masterton, Flanagan, Berkovic, \& Jackson, 2012; Curwood et al., 2015; Li et al., 2015; Luo et al., 2014; Tosun, Siddarth, Toga, Hermann, \& Caplan, 2011; Zhang et al., 2014), attention (Berman et al., 2010; Killory et al., 2011; Li et al., 2015; Luo et al., 2011; Luo et al., 2014; Zhang et al., 2014) and expressive language (Bai et al., 2011; Caplan et al., 2009; Curwood et al., 2015; Holmes, Brown, \& Tucker, 2004; Luo et al., 2011; Tosun et al., 2011; Tucker, Brown, Luu, \& Holmes, 2007).

\section{Limitations}

We opted to use single-arm meta-analyses and/or a metaanalysis of the difference between cases and controls.
This was chosen as sampling error may be of greater influence in a relatively small sample of the normal population, than it would be to estimate the true mean in AE, as a larger proportion of the group is likely to be included in a given geographical area. In addition, some studies did not match a control group specifically to children with $\mathrm{AE}$ as other types of epilepsy were also included. Therefore, residual confounding may still be present in those studies where there is a large age difference between patients with $\mathrm{AE}$ and controls. Some studies may have included patients with AE, which may have concomitant features, such as myoclonic jerks or atypical features. Also, some of the included studies focused on multiple epilepsy groups and therefore the findings of sub-analyses in patients with AE were often underpowered. Furthermore, some studies were relatively small in sample size and may be subject to a small-study effect. Importantly, multiple comparisons may have yielded false-positive findings (type 1 errors) in the included studies. Furthermore, the Flynn effect, which is a tendency of IQ to rise by $\sim 3$ points per decade on average, may have a small but significant bearing on our results (Trahan, Stuebing, Fletcher, \& Hiscock, 2014). Unfortunately, inconsistent reporting of neuropsychological test results across studies hindered comparison between studies and pooling of results. Granting that cognitive domains may overlap, assigning neuropsychological tests to a specific cognitive domain may lead to an oversimplified view.

\section{Future Work}

Future work should focus on large (multi-center) long-term observational studies to assess cognitive development and to identify children with AE most at risk for neuropsychological co-morbidity. Importantly, this may clarify whether repeated neuropsychological tests could be helpful and how cognitive development differs from healthy peers. Emphasis should be made to characterize children with AE according to semiology, EEG characteristics, and epilepsy syndrome. Especially separate reporting in JAE is lacking. Early identification of children with concomitant cognitive dysfunctions may help health care workers to initiate additional supportive programs, as well as communicate vulnerabilities and discuss teaching strategies with their school. A broad neuropsychological test battery, focusing on executive functioning, attention and verbal abilities should be used. Ideally, this should ideally be done before the start of anti-epileptic drugs and repeated at least once to track cognitive development. Given that cognitive dysfunctions may be case-specific, personalized supportive programs may be most useful, which may be discussed in a multidisciplinary team, consisting of at least a neurologist, neuropsychologists and a school expert. 
Acknowledgements We would like to thank I. Gijselhart (librarian at Epilepsy Centre Kempenhaeghe) for her assistance in building the search strategy and retrieving full-text articles.

Funding Information This research was made possible by collaborative funding by the MUMC+ and Epilepsy Center Kempenhaeghe.

\section{Compliance with Ethical Standards}

Conflict of Interest The authors report that they do not have any conflict of interest.

Open Access This article is distributed under the terms of the Creative Commons Attribution 4.0 International License (http:// creativecommons.org/licenses/by/4.0/), which permits unrestricted use, distribution, and reproduction in any medium, provided you give appropriate credit to the original author(s) and the source, provide a link to the Creative Commons license, and indicate if changes were made.

\section{References}

Adie, W. J. (1924). Pyknolepsy; a form of epilepsy in children, with a good prognosis. Proceedings of the Royal Society of Medicine, 17(Neurol Sect), 19-25.

Aldenkamp, A., \& Arends, J. (2004). The relative influence of epileptic EEG discharges, short nonconvulsive seizures, and type of epilepsy on cognitive function. Epilepsia, 45(1), 54-63.

Bai, X., Guo, J., Killory, B., Vestal, M., Berman, R., Negishi, M., et al. (2011). Resting functional connectivity between the hemispheres in childhood absence epilepsy. Neurology, 76(23), 1960-1967. https:// doi.org/10.1212/WNL.0b013e31821e54de

Baron, I. S. (2004). Neuropsychological evaluation of the child: Oxford University press.

Berg, A. T., Caplan, R., \& Hesdorffer, D. C. (2011). Psychiatric and neurodevelopmental disorders in childhood-onset epilepsy. Epilepsy \& Behavior, 20(3), 550-555. https://doi.org/10.1016/j. yebeh.2010.12.038

Berg, A. T., Levy, S. R., Testa, F. M., \& Blumenfeld, H. (2014). Longterm seizure remission in childhood absence epilepsy: Might initial treatment matter? Epilepsia, 55(4), 551-557. https://doi.org/10. 1111/epi.12551

Berman, R., Negishi, M., Vestal, M., Spann, M., Chung, M. H., Bai, X., et al. (2010). Simultaneous EEG, fMRI, and behavior in typical childhood absence seizures. Epilepsia, 51(10), 2011-2022. https:// doi.org/10.1111/j.1528-1167.2010.02652.x

Borenstein, M., Higgins, J. P., Hedges, L. V., \& Rothstein, H. R. (2017). Basics of meta-analysis: I(2) is not an absolute measure of heterogeneity. Research Synthesis Methods, 8(1), 5-18. https://doi.org/10. $1002 / \mathrm{jrsm} .1230$

Bouma, P. A., Westendorp, R. G., van Dijk, J. G., Peters, A. C., \& Brouwer, O. F. (1996). The outcome of absence epilepsy: A metaanalysis. Neurology, 47(3), 802-808.

Camfield, C. S., \& Camfield, P. R. (2007). Long-term social outcomes for children with epilepsy. Epilepsia, 48, 3-5. https://doi.org/10.1111/j. 1528-1167.2007.01390.x

Caplan, R., Levitt, J., Siddarth, P., Wu, K. N., Gurbani, S., Sankar, R., et al. (2009). Frontal and temporal volumes in childhood absence epilepsy. Epilepsia, 50(11), 2466-2472. https://doi.org/10.1111/j. 1528-1167.2009.02198.x

Caplan, R., Siddarth, P., Stahl, L., Lanphier, E., Vona, P., Gurbani, S., et al. (2008). Childhood absence epilepsy: Behavioral, cognitive, and linguistic comorbidities. Epilepsia, 49(11), 1838-1846. https:// doi.org/10.1111/j.1528-1167.2008.01680.x
Carney, P. W., Masterton, R. A., Flanagan, D., Berkovic, S. F., \& Jackson, G. D. (2012). The frontal lobe in absence epilepsy: EEG-fMRI findings. Neurology, 78(15), 1157-1165. https://doi.org/10.1212/WNL. 0b013e31824f801d

Cerminara, C., D’Agati, E., Casarelli, L., Kaunzinger, I., Lange, K. W., Pitzianti, M., et al. (2013). Attention impairment in childhood absence epilepsy: An impulsivity problem? Epilepsy \& Behavior, 27(2), 337-341. https://doi.org/10.1016/j.yebeh.2013.02.022

Cheng, D., Yan, X., Gao, Z., Xu, K., Zhou, X., \& Chen, Q. (2017). Common and distinctive patterns of cognitive dysfunction in children with benign epilepsy syndromes. Pediatr Neurol, 72, 3641.e31. https://doi.org/10.1016/j.pediatrneurol.2016.12.005

Cnaan, A., Shinnar, S., Arya, R., Adamson, P. C., Clark, P. O., Dlugos, D., et al. (2017). Second monotherapy in childhood absence epilepsy. Neurology, 88(2), 182-190. https://doi.org/10.1212/wnl. 0000000000003480

Conant, L. L., Wilfong, A., Inglese, C., \& Schwarte, A. (2010). Dysfunction of executive and related processes in childhood absence epilepsy. Epilepsy \& Behavior, 18(4), 414-423. https://doi. org/10.1016/j.yebeh.2010.05.010

Conde-Guzon, P. A., \& Cancho-Candela, R. (2012). Idiopathic generalized epilepsies with absence seizures with valproic acid treatment: Neuropsychological disorders. Revista de Neurologia, 55(2), 65-73.

Covanis, A., Skiadas, K., Loli, N., Lada, C., \& Theodorou, V. (1992). Absence epilepsy: Early prognostic signs. Seizure, 1(4), 281-289.

Currier, R. D., Kooi, K. A., \& Saidman, L. J. (1963). Prognosis of "pure" petit mal; a follow-up study. Neurology, 13, 959-967.

Curwood, E. K., Pedersen, M., Carney, P. W., Berg, A. T., Abbott, D. F., \& Jackson, G. D. (2015). Abnormal cortical thickness connectivity persists in childhood absence epilepsy. Annals of Clinical Translational Neurology, 2(5), 456-464. https://doi.org/10.1002/ acn3.178

D’Agati, E., Cerminara, C., Casarelli, L., Pitzianti, M., \& Curatolo, P. (2012). Attention and executive functions profile in childhood absence epilepsy. Brain Dev, 34(10), 812-817. https://doi.org/10. 1016/j.braindev.2012.03.001

Dlugos, D., Shinnar, S., Cnaan, A., Hu, F., Moshe, S., Mizrahi, E., et al. (2013). Pretreatment EEG in childhood absence epilepsy: Associations with attention and treatment outcome. Neurology, 81(2), 150-156. https://doi.org/10.1212/WNL.0b013e31829a3373

Fastenau, P. S., Johnson, C. S., Perkins, S. M., Byars, A. W., deGrauw, T. J., Austin, J. K., et al. (2009). Neuropsychological status at seizure onset in children: Risk factors for early cognitive deficits. Neurology, 73(7), 526-534. https://doi.org/10.1212/WNL. 0b013e3181b23551

Franzoni, E., Matricardi, S., Di Pisa, V., Capovilla, G., Romeo, A., Tozzi, E., et al. (2015). Refractory absence seizures: An Italian multicenter retrospective study. European Journal of Paediatric Neurology, 19(6), 660-664. https://doi.org/10.1016/j.ejpn.2015.07.008

Gencpinar, P., Kalay, Z., Turgut, S., Bozkurt, O., Duman, O., Ozel, D., et al. (2016). Evaluation of executive functions in patients with childhood absence epilepsy. Journal of Child Neurology, 31(7), 824-830. https://doi.org/10.1177/0883073815623632

Glauser, T. A., Cnaan, A., Shinnar, S., Hirtz, D. G., Dlugos, D., Masur, D., et al. (2010). Ethosuximide, valproic acid, and lamotrigine in childhood absence epilepsy. The New England Journal of Medicine, 362(9), 790-799. https://doi.org/10.1056/ NEJMoa0902014

Glauser, T. A., Cnaan, A., Shinnar, S., Hirtz, D. G., Dlugos, D., Masur, D., et al. (2013). Ethosuximide, valproic acid, and lamotrigine in childhood absence epilepsy: Initial monotherapy outcomes at 12 months. Epilepsia, 54(1), 141-155. https://doi.org/10.1111/epi. 12028

Guerrini, R. (2006). Epilepsy in children. Lancet, 367(9509), 499-524. https://doi.org/10.1016/S0140-6736(06)68182-8 
Guerrini, R., Melani, F., Brancati, C., Ferrari, A. R., Brovedani, P., Biggeri, A., et al. (2015). Dysgraphia as a mild expression of dystonia in children with absence epilepsy. PLoS One, 10(7), e0130883. https://doi.org/10.1371/journal.pone.0130883

Henkin, Y., Kishon-Rabin, L., Pratt, H., Kivity, S., Sadeh, M., \& Gadoth, N. (2003). Linguistic processing in idiopathic generalized epilepsy: An auditory event-related potential study. Epilepsia, 44(9), 1207-1217.

Henkin, Y., Sadeh, M., Kivity, S., Shabtai, E., Kishon-Rabin, L., \& Gadoth, N. (2005). Cognitive function in idiopathic generalized epilepsy of childhood. Developmental Medicine and Child Neurology, 47(2), 126-132.

Holmes, M. D., Brown, M., \& Tucker, D. M. (2004). Are "generalized" seizures truly generalized? Evidence of localized mesial frontal and frontopolar discharges in absence. Epilepsia, 45(12), 1568-1579. https://doi.org/10.1111/j.0013-9580.2004.23204.x

Holtkamp, M., Senf, P., Kirschbaum, A., \& Janz, D. (2014). Psychosocial long-term outcome in juvenile myoclonic epilepsy. Epilepsia, 55(11), 1732-1738. https://doi.org/10.1111/epi.12751

Hommet, C., Billard, C., Motte, J., Passage, G. D., Perrier, D., Gillet, P., et al. (2001). Cognitive function in adolescents and young adults in complete remission from benign childhood epilepsy with centrotemporal spikes. Epileptic Disorders, 3(4), 207-216.

Hughes, J. R. (2009). Absence seizures: A review of recent reports with new concepts. Epilepsy \& Behavior, 15(4), 404-412. https://doi.org/ 10.1016/j.yebeh.2009.06.007

IJff, D. M., van Veenendaal, T. M., Debeij-van Hall, M. H., Jansen, J. F., de Louw, A. J., Majoie, M. H., et al. (2016). The cognitive profile of Ethosuximide in children. Paediatric Drugs, 18(5), 379-385. https://doi.org/10.1007/s40272-016-0187-z

Jafarian, M., Karimzadeh, F., Alipour, F., Attari, F., Lotfinia, A. A., Speckmann, E. J., ... Gorji, A. (2015). Cognitive impairments and neuronal injury in different brain regions of a genetic rat model of absence epilepsy. Neuroscience, 298, 161-170. https://doi.org/10. 1016/j.neuroscience.2015.04.033

Kernan, C. L., Asarnow, R., Siddarth, P., Gurbani, S., Lanphier, E. K., Sankar, R., et al. (2012). Neurocognitive profiles in children with epilepsy. Epilepsia, 53(12), 2156-2163. https://doi.org/10.1111/j. 1528-1167.2012.03706.x

Killory, B. D., Bai, X., Negishi, M., Vega, C., Spann, M. N., Vestal, M., et al. (2011). Impaired attention and network connectivity in childhood absence epilepsy. Neuroimage, 56(4), 2209-2217. https://doi. org/10.1016/j.neuroimage.2011.03.036

Lenck-Santini, P. P., \& Scott, R. C. (2015). Mechanisms responsible for cognitive impairment in epilepsy. Cold Spring Harbor Perspectives in Medicine, 5(10). https://doi.org/10.1101/cshperspect.a022772

Levav, M., Mirsky, A. F., Herault, J., Xiong, L., Amir, N., \& Andermann, E. (2002). Familial association of neuropsychological traits in patients with generalized and partial seizure disorders. Journal of Clinical and Experimental Neuropsychology, 24(3), 311-326. https://doi.org/10.1076/jcen.24.3.311.985

Li, Q., Cao, W., Liao, X., Chen, Z., Yang, T., Gong, Q., et al. (2015). Altered resting state functional network connectivity in children absence epilepsy. Journal of the Neurological Sciences, 354(1-2), 79-85. https://doi.org/10.1016/j.jns.2015.04.054

Lopes, A. F., Monteiro, J. P., Fonseca, M. J., Robalo, C., \& Simoes, M. R. (2014). Memory functioning in children with epilepsy: Frontal lobe epilepsy, childhood absence epilepsy, and benign epilepsy with centrotemporal spikes. Behavioural Neurology, 2014, 218637. https://doi.org/10.1155/2014/218637

Lopes, A. F., Simoes, M. R., Monteiro, J. P., Fonseca, M. J., Martins, C., Ventosa, L., et al. (2013). Intellectual functioning in children with epilepsy: Frontal lobe epilepsy, childhood absence epilepsy and benign epilepsy with centro-temporal spikes. Seizure, 22(10), 886892. https://doi.org/10.1016/j.seizure.2013.08.002
Loughman, A., Bendrups, N. A., \& D'Souza, W. J. (2016). A systematic review of psychiatric and psychosocial comorbidities of genetic generalised epilepsies (GGE). Neuropsychology Review, 26(4), 364-375. https://doi.org/10.1007/s11065-016-9333-1

Loughman, A., Bowden, S. C., \& D'Souza, W. (2014). Cognitive functioning in idiopathic generalised epilepsies: A systematic review and meta-analysis. Neuroscience and Biobehavioral Reviews, 43, 20 34. https://doi.org/10.1016/j.neubiorev.2014.02.012

Loughman, A., Bowden, S. C., \& D'Souza, W. J. (2017). A comprehensive assessment of cognitive function in the common genetic generalized epilepsy syndromes. European Journal of Neurology, 24(3), 453-460. https://doi.org/10.1111/ene.13232

Luo, C., Li, Q., Lai, Y., Xia, Y., Qin, Y., Liao, W., et al. (2011). Altered functional connectivity in default mode network in absence epilepsy: A resting-state fMRI study. Human Brain Mapping, 32(3), 438 449. https://doi.org/10.1002/hbm.21034

Luo, C., Yang, T., Tu, S., Deng, J., Liu, D., Li, Q., et al. (2014). Altered intrinsic functional connectivity of the salience network in childhood absence epilepsy. Journal of the Neurological Sciences, 339(1-2), 189-195. https://doi.org/10.1016/j.jns.2014.02.016

Marengoni, A., Vetrano, D. L., Manes-Gravina, E., Bernabei, R., Onder, G., \& Palmer, K. (2018). The relationship between COPD and frailty: A systematic review and Meta-analysis of observational studies. Chest, 154(1), 21-40. https://doi.org/10.1016/j.chest.2018.02.014

Martinez-Ferrandez, C., Martinez-Salcedo, E., Casas-Fernandez, C., Alarcon-Martinez, H., Ibanez-Mico, S., \& Domingo-Jimenez, R. (2017). Long-term prognosis of childhood absence epilepsy. Neurologia. https://doi.org/10.1016/j.nrl.2016.12.005

Masur, D., Shinnar, S., Cnaan, A., Shinnar, R. C., Clark, P., Wang, J., et al. (2013). Pretreatment cognitive deficits and treatment effects on attention in childhood absence epilepsy. Neurology, 81(18), 15721580. https://doi.org/10.1212/WNL.0b013e3182a9f3ca

Moher, D., Liberati, A., Tetzlaff, J., \& Altman, D. G. (2009). Preferred reporting items for systematic reviews and meta-analyses: The PRISMA statement. Journal of Clinical Epidemiology, 62(10), 1006-1012. https://doi.org/10.1016/j.jclinepi.2009.06.005

Mostafa, M., Talaat, F., Shalaby, N., El-Fayoumy, N., \& Labib, D. (2014). Cognitive impairment in idiopathic generalized epilepsies. Egyptian Journal of Neurology, Psychiatry and Neurosurgery, 51(2), 215-221.

Myers, T., \& Fecske, E. (2016). The "staring" child. The Nurse Practitioner, 41(1), 9-11. https://doi.org/10.1097/01.NPR. $0000460860.75481 .8 \mathrm{a}$

Nicolai, J., Ebus, S., Biemans, D. P., Arends, J., Hendriksen, J., Vles, J. S., \& Aldenkamp, A. P. (2012). The cognitive effects of interictal epileptiform EEG discharges and short nonconvulsive epileptic seizures. Epilepsia, 53(6), 1051-1059. https://doi.org/10.1111/j.15281167.2012.03491.x

Nolan, M. A., Redoblado, M. A., Lah, S., Sabaz, M., Lawson, J. A., Cunningham, A. M., et al. (2004). Memory function in childhood epilepsy syndromes. Journal of Paediatrics and Child Health, 40(12), 20-27.

Oostrom, K. J., Smeets-Schouten, A., Kruitwagen, C., Peters, A. C. B., Jennekens-Schinkel, A., \& DuSech. (2003). Not only a matter of epilepsy: Early problems of cognition and behavior in children with "epilepsy only" - a prospective, longitudinal, controlled study starting at diagnosis. Pediatrics, 112(6), 1338-1344. https://doi. org/10.1542/peds.112.6.1338

Panayiotopoulos, C. P. (2001). Treatment of typical absence seizures and related epileptic syndromes. Paediatric Drugs, 3(5), 379-403.

Pavone, P., Bianchini, R., Trifiletti, R. R., Incorpora, G., Pavone, A., \& Parano, E. (2001). Neuropsychological assessment in children with absence epilepsy. Neurology, 56(8), 1047-1051.

Schraegle, W. A., Nussbaum, N. L., \& Stefanatos, A. K. (2016). Listlearning and verbal memory profiles in childhood epilepsy 
syndromes. Epilepsy \& Behavior, 62, 159-165. https://doi.org/10. 1016/j.yebeh.2016.07.021

Schwarzer, G. (2007). Meta: An R package for meta-analysis. $R$ news, $7(3), 40-45$.

Shinnar, R. C., Shinnar, S., Cnaan, A., Clark, P., Dlugos, D., Hirtz, D. G., et al. (2017). Pretreatment behavior and subsequent medication effects in childhood absence epilepsy. Neurology. https://doi.org/10. 1212/wnl.0000000000004514

Shuster, J. J. (2011). Cochrane handbook for systematic reviews for interventions, version 5.1. 0, published 3/2011. Julian PT Higgins and Sally green, editors. Research Synthesis Methods, 2(2), 126-130.

Sinclair, D. B., \& Unwala, H. (2007). Absence epilepsy in childhood: Electroencephalography (EEG) does not predict outcome. Journal of Child Neurology, 22(7), 799-802. https://doi.org/10.1177/ 0883073807304198

Siren, A., Kylliainen, A., Tenhunen, M., Hirvonen, K., Riita, T., \& Koivikko, M. (2007). Beneficial effects of antiepileptic medication on absence seizures and cognitive functioning in children. Epilepsy \& Behavior, 11(1), 85-91. https://doi.org/10.1016/j. yebeh.2007.04.009

Talero-Gutierrez, C., Sanchez-Torres, J. M., \& Velez-van-Meerbeke, A. (2015). Learning skills and academic performance in children and adolescents with absence epilepsy. Neurologia, 30(2), 71-76. https://doi.org/10.1016/j.nrl.2013.10.011

Tenney, J. R., \& Glauser, T. A. (2013). The current state of absence epilepsy: Can we have your attention? Epilepsy Curr, 13(3), 135140. https://doi.org/10.5698/1535-7511-13.3.135

Thompson, S. G., \& Higgins, J. P. (2002). How should meta-regression analyses be undertaken and interpreted? Statistics in Medicine, 21(11), 1559-1573. https://doi.org/10.1002/sim.1187

Tosun, D., Siddarth, P., Toga, A. W., Hermann, B., \& Caplan, R. (2011). Effects of childhood absence epilepsy on associations between regional cortical morphometry and aging and cognitive abilities. Human Brain Mapping, 32(4), 580-591. https://doi.org/10.1002/ hbm. 21045

Trahan, L. H., Stuebing, K. K., Fletcher, J. M., \& Hiscock, M. (2014). The Flynn effect: A meta-analysis. Psychological Bulletin, 140(5), 1332-1360. https://doi.org/10.1037/a0037173
Trinka, E., Baumgartner, S., Unterberger, I., Unterrainer, J., Luef, G., Haberlandt, E., \& Bauer, G. (2004). Long-term prognosis for childhood and juvenile absence epilepsy. Journal of Neurology, 251(10), 1235-1241. https://doi.org/10.1007/s00415-004-0521-1

Tucker, D. M., Brown, M., Luu, P., \& Holmes, M. D. (2007). Discharges in ventromedial frontal cortex during absence spells. Epilepsy \& Behavior, 11(4), 546-557.

Urena-Hornos, T., Rubio-Rubio, R., Gros-Esteban, D., Cabrerizo de Diago, R., Pena-Segura, J. L., \& Lopez-Pison, J. (2004). Absence epilepsy. A review of our 14 years' experience. Revista de Neurologia, 39(12), 1113-1119.

Vanasse, C. M., Beland, R., Carmant, L., \& Lassonde, M. (2005). Impact of childhood epilepsy on reading and phonological processing abilities. Epilepsy \& Behavior, 7(2), 288-296. https://doi.org/10.1016/j. yebeh.2005.05.008

Vega, C., Vestal, M., DeSalvo, M., Berman, R., Chung, M., Blumenfeld, H., \& Spann, M. N. (2010). Differentiation of attention-related problems in childhood absence epilepsy. Epilepsy \& Behavior, 19(1), 82-85. https://doi.org/10.1016/j.yebeh.2010.06.010

Verrotti, A., Olivieri, C., Agostinelli, S., Coppola, G., Parisi, P., Grosso, S., et al. (2011). Long term outcome in children affected by absence epilepsy with onset before the age of three years. Epilepsy \& Behavior, 20(2), 366-369. https://doi.org/10.1016/j.yebeh.2010. 12.015

Viechtbauer, W. (2010). Conducting meta-analyses in R with the metafor package. Journal of Statistical Software, 36(3), 1-48.

Wirrell, E. C., Camfield, C. S., Camfield, P. R., Gordon, K. E., \& Dooley, J. M. (1996). Long-term prognosis of typical childhood absence epilepsy: Remission or progression to juvenile myoclonic epilepsy. Neurology, 47(4), 912-918.

Zhang, Z., Liao, W., Wang, Z., Xu, Q., Yang, F., Mantini, D., et al (2014). Epileptic discharges specifically affect intrinsic connectivity networks during absence seizures. Journal of the Neurological Sciences, 336(1-2), 138-145. https://doi.org/10.1016/j.jns.2013. 10.024

Publisher's Note Springer Nature remains neutral with regard to jurisdictional claims in published maps and institutional affiliations. 